\title{
DUSP5 expression in left ventricular cardiomyocytes of young hearts regulates thyroid hormone (T3)-induced proliferative ERK1/2 signaling
}

\section{Nikolay Bogush}

Cardiology, Emory University School of Medicine

\section{Lin Tan}

Cardiology, Emory University School of Medicine

Hussain Naib

Cardiology, Emory University School of Medicine Ebrahim Faizullabhoy

Cardiology, Emory University School of Medicine John W. Calvert

Surgery, Emory University School of Medicine

Siiri E. lismaa

Victor Chang Cardiac Research Institute, Darlinghurst, New South Wales, Australia

\section{Ankan Gupta}

Developmental Vascular Biology Program, Division of Neonatology, Department of Pediatrics,

Department of Obstetrics and Gynecology, Children's Research Institute, Medical College of Wisconsin, Milwaukee, WI, USA

\section{Ramani Ramchandran}

Developmental Vascular Biology Program, Division of Neonatology, Department of Pediatrics, Department of Obstetrics and Gynecology, Children's Research Institute, Medical College of Wisconsin, Milwaukee, WI, USA

\section{David I.K. Martin}

Children's Hospital Oakland Research Institute, Oakland, CA, USA

\section{Robert M. Graham}

Victor Chang Cardiac Research Institute, Darlinghurst, New South Wales, Australia

\section{Ahsan Husain}

Cardiology, Emory University School of Medicine

\section{Nawazish Naqvi ( $\nabla$ nnaqvi@emory.edu )}

Cardiology, Emory University School of Medicine https://orcid.org/0000-0002-4760-8481 


\section{Research Article}

Keywords: Thyroid hormone, cell proliferation, DUSP5, ERK1/2 signaling, IGF-1/IGF-1R signaling, genetic lineage tracing

Posted Date: September 9th, 2020

DOI: https://doi.org/10.21203/rs.3.rs-73803/v1

License: (1) This work is licensed under a Creative Commons Attribution 4.0 International License.

Read Full License

Version of Record: A version of this preprint was published at Scientific Reports on December 1st, 2020. See the published version at https://doi.org/10.1038/s41598-020-78825-x. 


\section{DUSP5 expression in left ventricular cardiomyocytes of young hearts regulates thyroid hormone (T3)-induced proliferative ERK1/2 signaling}

Nikolay Bogush $1, \uparrow$, Lin Tan1, $\uparrow$, Hussain Naib1, Ebrahim Faizullabhoy1, John W. Calvert2, Siiri E. Iismaa3, Ankan Gupta4, Ramani Ramchandran4, David I.K. Martin5, Robert M. Graham3, Ahsan Husain $1,{ }^{*}$ and Nawazish Naqvi1,*

1Department of Medicine (Cardiology), Emory University School of Medicine, Atlanta, GA, USA. 2Department of Surgery, Carlyle Fraser Heart Center, Emory University School of Medicine, Atlanta, GA, USA. 3Victor Chang Cardiac Research Institute, Darlinghurst, New South Wales, Australia. 4Developmental Vascular Biology Program, Division of Neonatology, Department of Pediatrics, Department of Obstetrics and Gynecology, Children's Research Institute, Medical College of Wisconsin, Milwaukee, WI, USA. 5Children's Hospital Oakland Research Institute, Oakland, CA, USA.

$\uparrow$ These authors contributed equally: Nikolay Bogush, Lin Tan.

*Corresponding Authors: Nawazish Naqvi, PhD, Department of Medicine (Cardiology), Emory University, 323 WMRB, 101 Woodruff Circle, Atlanta Georgia 30322. E-mail nnaqvi@emory.edu or Ahsan Husain, PhD, Department of Medicine (Cardiology), Emory University, 3311 WMRB, 101 Woodruff Circle, Atlanta Georgia 30322. Tel: 404-727-8125; Emailahusai2@emory.edu

Short title: Spatial heterogeneity of T3-proliferative signaling in young hearts.

Key words: Thyroid hormone, cell proliferation, DUSP5, ERK1/2 signaling, IGF-1/IGF-1R signaling, genetic lineage tracing.

Word Count: Abstract 199; Main Text 4,062; Methods 1,687 


\begin{abstract}
A developmental surge in thyroid hormone (T3) after postnatal day-10 (P10), in mice, results in a burst of cardiomyocyte proliferation. This finding remains controversial, which could arise from perceived homogeneity of myocardial sampling for immunoblotting and immunohistochemical studies, or from differences in enzymatic digestion efficiency for cardiomyocyte isolation used to determine total ventricular cardiomyocyte numbers. Using a highly efficient (>97\%) method for cardiomyocyte isolation, we show that exogenous T3 administered in vivo to post-neonatal (after postnatal P6) mice increased cardiomyocyte numbers. Using S- and M-phase markers, and lineage-tracing tools to assay for cell cycle activation and cytokinesis, respectively, we show that the T3-mediated increase in cardiomyocytes is confined to cells of the left ventricular (LV) apex; such spatial heterogeneity also being observed during normal development, which might confound substantiation of developmental increases in cardiomyocyte proliferation. In these apical cardiomyocytes, T3 stimulates proliferative ERK1/2 signaling, whereas those in the LV base are tonically inhibited by expression of the nuclear phospho-ERK1/2-specific dual-specificity phosphatase, DUSP5. In P21 Dusp5-^- mice, cardiomyocyte endowment and LV mass are increased relative to agematched wild-type controls, suggesting that DUSP5 regulates early heart growth. Identification of mechanisms regulating cardiomyocyte proliferation may allow development of cardiac regenerative therapies.
\end{abstract}




\section{Introduction}

The rodent heart grows rapidly after birth; quadrupling in size over a period of just a few weeks . A key feature of this growth is an increase in stroke volume1, commensurate with the rapid expansion of the circulatory system during postnatal development. As the newborn heart grows its constituent cardiomyocytes mature. The increase in the mass of the left ventricle (LV) and the fetal-to-adult shift in gene expression patterns (e.g., increased postnatal expression of Mhy6, the gene encoding $\alpha$-myosin heavy chain)2 increase LV contractile performance1.

However, the question of how muscle mass, particularly that of the LV, increases during this developmental period remains controversial. As the young hearts grows, cardiomyocytes hypertrophy due to marked elongation with smaller increases in widths1. What remains controversial is the extent to which rodent cardiomyocytes also increase in numbers in early postnatal life. By the end of the 1990's, two views had emerged: first, that murine cardiomyocyte proliferation ceases immediately before birth and that any cell cycle activity observed postnatally results only in increased nuclear ploidy and nucleation state (e.g., mono-, bi- and multinuclear)3; and, second, that cardiomyocyte numbers also increase after birth, within the first 3 weeks of life; this increase being linked to the rate of body growth4. Using a variety of approaches, several studies have shown that between a few days after birth and postnatal day 7 (P7) there is a $30 \%$ to $60 \%$ increase in cardiomyocyte numbers $1,5,6$. Whether cardiomyocyte numbers in murine hearts increase substantively after this time continues to be debated and has been the subject of multiple reports as well as reviews and editorials 1,6-9.

The controversy began with a paper published in Cell by our group that reported an approximately $30 \%$ increase in ventricular cardiomyocyte numbers between the end of the second and the beginning of the third week of life. We also reported that the mechanism of this 
increase was a developmental rise in the levels of the thyroid hormone, T3, in the circulation. Although some had also observed increases in murine cardiomyocytes during this postnatal developmental window (cf. ref 10 and also discussion in ref 7) and cardiomyocyte proliferation in humans has been shown to contribute to myocardial growth during the first 20 years of life11, others who sought to confirm our findings reported negative results6,12,13. These studies included sequential assessments of $\mathrm{S}$ phase and mitosis as well as cardiomyocyte number assessments using the emerging method of design-based stereology. Such diametrically opposite views on the mechanism and timing of early heart growth are not confined to rodents, but also extend to disparate findings regarding postnatal cardiomyocyte proliferative responses in humans11,14.

Here we identify potential biological origins of variability that contribute towards discordance among laboratories on the above issues thus advancing our understanding about early postnatal heart growth. An important issue is keeping consistent animal husbandry conditions during these developmental studies7 as it impacts circulating T3 levels which impacts cardiomyocyte proliferation in the post-neonatal (after P6) period1. At birth, T3 levels in the circulation are virtually undetectable. The major postnatal increase in T3, in mice, occurs around P10, which results in circulating levels similar to those found in adults1. The early postnatal regulation of circulating T3 is complex but is intimately linked to mouse growth. This is potentially of considerable importance, since significant differences exist between natural litter sizes and body weight growth of individual pups; pups in smaller litters grow faster and attain higher body weights than in larger litters. A connection between body growth and postnatal increase in cardiomyocyte numbers was reported several years ago by Bai et al. (1990)4. They showed that, at P21, those rats that were "slow growing" (12 pups/litter) had 20\% fewer cardiomyocytes than "fast growing" rats (4 pups/litter). These concerns led us to question 
whether differences in growth rates, and therefore the timing and robustness of the endogenous surge of T3 during preadolescence, could have been an important source of variability in recent reports on postnatal cardiomyocyte proliferation7.

A further consideration with respect to postnatal cardiomyocyte proliferative responses is the uniformity of cardiomyocytes throughout the ventricles of the preadolescent mouse heart, and, as a corollary, of their responses to T3. To date, uniformity has been assumed by virtually all investigators, but has never been tested.

Here, we evaluated if exogenous administration of $\mathrm{T} 3$ can activate cardiomyocyte proliferation in post-P6 hearts (that is, immediately after the neonatal period). In these studies, we controlled for litter size to minimize any natural variability in body growth and associated cardiomyocyte proliferation, and we focused on the developmental period just before endogenous T3 levels increase, so as not to complicate the effects of exogenous T3 administration with those of endogenous T3. Importantly, also, we critically examined if exogenous T3 can differentially stimulate proliferation in ventricular cardiomyocytes depending on their location within the heart. We show that T3 stimulates post-P6 cardiomyocytes to proliferate, albeit in a spatially-restricted manner, with only cells of the LV apex responding; a finding that could shed light on the failure of other investigators to replicate our initial findings. Moreover, our studies reveal the molecular mechanism distinguishing cardiomyocytes in the apex that proliferate in response to $\mathrm{T} 3$, from those in the base that do not. This involves the expression of DUSP5, a nuclear phospho-ERK1/2-specific dual specificity phosphatase that limits availability of the mitogenic mediator, phosphorylated ERK1/2, in the nucleus; DUSP5 expression being developmentally regulated and apparent initially in cardiomyocytes in the base, but only much later in LV apical cells. 


\section{Results}

\section{LV apical cardiomyocytes of post-P6 hearts proliferate in response to exogenous $\mathrm{T3}$. To} determine if post-P6 ventricular cardiomyocytes retain proliferative capacity, we administered T3 daily (3.5 ng/g, daily, i. p.), from P7 to P9. At P12, hearts were enzymatically disaggregated and cell suspensions created from the cardiac ventricles. Digestion efficiencies were $98 \%$ (97.9 $\pm 1.1 \%$ and $98.6 \pm 0.21 \%$ in vehicle- and T3-treated hearts, respectively; $P=0.53 ; n=4$ ). Cardiomyocytes in these cell suspensions, identified by their size and by rod shape, were counted using a hemocytometer. In agreement with our previous in vivo findings in neonatal cardiomyocytes15, exogenous $\mathrm{T} 3$ administration beginning at P7 increased ventricular cardiomyocyte numbers by $\sim 18 \%(P<0.001)$ (Fig. 1A).

We then sought to determine if the proliferative response to $\mathrm{T} 3$ occurs uniformly in all $\mathrm{LV}$ cardiomyocytes. Initially, we evaluated cyclins D1, A2 and B1 responses to T3; these cyclins promote G1/S phase transition, S phase, and G2/M phase transition, respectively. Forty hours after an in vivo T3 challenge in P8 mice, expression of cyclins D1, A2 and B1 was increased only in the LV (Fig. 1B) and, within the LV, these cyclins were primarily expressed in myocardial tissue of the apex relative to that of the base $(P<0.001)$ (Fig. 1C).

Next, we studied whether cardiomyocytes of the P8 LV apex and the LV base respond differentially to exogenous $\mathrm{T} 3$, as implied by our tissue cyclin expression studies. We examined cell cycle reentry by 5 -ethynyl-2'-deoxyuridine (EdU) incorporation. We also assayed mitosis by the presence of phosphorylated histone-3 $(\mathrm{pH} 3)$. Administration of $\mathrm{T} 3$ increased EdU and $\mathrm{pH} 3$ labeling in cardiomyocytes of the LV apex (Fig. 2A,B), with cell cycle entry and mitosis being observed in both mono- and binucleated LV apical cardiomyocytes (Fig. 2B). While cell cycle entry was seen in cardiomyocytes derived from both the LV apex and base, T3-stimulated 
mitosis was evident only in apical cardiomyocytes (Fig. 2A,B).

To confirm that T3-stimulated cardiomyocyte replication is indeed limited to cells in the LV apex, we used a genetic lineage tracing approach. Specifically, we evaluated the proliferative strategies of individual cardiomyocytes of the LV apex relative to those of the base using multicolor clonal analysis of cardiomyocytes in double transgenic Myh6-MerCreMer::Rosa26fsConfetti mice16. In this double transgenic model, limited Cre activation (by administration of 10 $\mathrm{mg} / \mathrm{kg}$ 4-hydroxytamoxifen to P6 mice) resulted in cardiomyocytes being labeled with either RFP, YFP, CFP or GFP. The frequency of RFP and YFP labeling events was $<1.5 \%$, each, while cardiomyocytes labeled with membrane-targeted CFP and nuclear-targeted GFP were much more infrequent. By contrast, at higher 4-hydroxytamoxifen doses, a greater percentage of cardiomyocytes were CFP-labeled and bichromatic cardiomyocytes were also observed (data not shown). In subsequent studies with $\mathrm{T} 3$ administration to $\sim \mathrm{P} 8$ mice, we relied on quantifying monochromatic cluster frequencies of RFP- and YFP-labeled cardiomyocytes after activation of Cre expression using $10 \mu \mathrm{g} / \mathrm{g}$ 4-hydroxytamoxifen.

In 4-hydroxytamoxifen-pretreated Myh6-MerCreMer::Rosa26fs-Confetti mice, T3, given daily over 3 days from $\mathrm{P} 7$ to $\mathrm{P} 9$, increased the relative frequency of monochromatic cardiomyocyte clusters by $\sim 6.5$-fold in the LV apex relative to its base $(P=0.005)$ (Fig. 2 C). The majority of monochromatic cardiomyocyte clusters in the LV apex consisted of 2 cells (Fig. 2C), and were distributed throughout the tissue section, indicating that cardiomyocyte proliferation resulted from multiple individual cell replication events rather than clonal expansion of a few highly proliferative cells. In addition, monochromatic clusters of $>2$ cells were observed in the LV apex, but never in the LV base. These studies indicate that T3stimulated cardiomyocyte proliferation in early post-P6 LVs is spatially restricted to the apex. 


\section{Spatial heterogeneity of proliferative signaling in the developing postnatal heart. Our}

earlier studies, using pups from litters of $\sim 7 /$ dam showed that a substantial surge in circulating T3 levels that occurs developmentally from P12, is associated with a transient induction of cardiomyocyte proliferation during mid-preadolescence that is at P15 1 . We also show that this developmental phase of cardiomyocyte proliferation can be blocked by inhibiting T3 biosynthesis with propylthiouracil (PTU) given from P7 1. Thus, we next tested whether the response to exogenous $\mathrm{T} 3$ is restricted to cardiomyocytes of the $\mathrm{LV}$ apex and is associated with maturation of the heart during this critical developmental window. Mitogenic stimuli elicit sustained increases in phosphorylated (p)-ERK1/2 that in turn activate multiple gene programs, resulting in effector proteins synthesis (such as cyclins D1, A2) and cell proliferation17,18. Here, we used p-ERK1/2 and cyclin A2 expression as surrogates for proliferative signaling. Also, we examined if this apex-restricted proliferative signaling phenotype is dependent on the developmental surge in circulating T3 during the second week of life.

Suckling of pups impacts on the timing of the natural surge that occurs in circulating T3 levels during preadolescence19. Adequate supply of milk for each mouse and therefore litter size is also known to impact cardiomyocyte number during the first 3-weeks after birth4. To minimize natural intra-litter variation in circulating T3 levels and body growth during preadolescence, we adjusted litter sizes to 4 pups/dam at P2 so that every pup could access a teat and have an adequate supply of milk. As shown in figure 3A, circulating T3 levels rise from P10 to P14 in pups from litters of $4 /$ dam, as we previously reported in pups from litters of $\sim 7 /$ dam 1 , however, in the former, the developmental surge occurred 2-days earlier and the levels of circulating T3 during preadolescence were significantly higher.

Evaluation of cyclin A2 expression in the postnatal period, revealed that it is transiently 
induced during the neonatal period starting at P4, with peak cyclin A2 levels at P5 that then wane by the end of neonatal period (P6) (Fig. 3B). After a brief quiescence, during the early post-P6 period, the expression of cell cycle promoting cyclin A2 increased significantly in the LV apex but not LV base, starting at P12, peaking at P13 and then declining after P15 to undetectable levels (Fig. 3B). While cyclin A2+cardiomyocytes in the P13 LV apex were readily detectible by immunohistochemical staining (Fig. 3C), we could not find any cyclin A2+cardiomyocyte in the P13 LV base (data not shown). To determine if this characteristic LV apex-restricted proliferative signaling of the preadolescent heart is dependent on developmental surge in circulating T3, we inhibited T3 biosynthesis by administrating PTU from P7 to P13, as previously reported. We show that cardiomyocytes from the LV apex but not the LV base of the preadolescent murine heart show increased cardiomyocyte expression of p-ERK1/2 and cyclin A2 - effects that are inhibited if we block T3 biosynthesis with PTU (Fig. 3D). Collectively, these studies show that during the second week of life cardiomyocytes in the developing murine LV are inherently heterogeneous in their response to developmental growth cues.

\section{DUSP5 inhibits T3-stimulated ERK1/2 phosphorylation in postnatal cardiomyocytes. T3-} stimulated proliferation of murine neonatal (P2) cardiomyocytes is mediated by IGF-1/IGF-1R activation15, which involves Ras/Raf/MAPK kinase (MEK)/ERK signaling17. MEK1/2 activates ERK1/2 by phosphorylating T202 and Y204 17, which is required for ERK1/2 translocation to the nucleus18. Sustained increases in p-ERK1/2 in the nucleus activate multiple transcription factors, ultimately resulting in effector protein synthesis and cell proliferation17. We tested whether, the difference in the proliferative capacity of cardiomyocytes in the P8 LV apex and base is due to differential regulation of IGF-1/IGF-1R/ERK1/2 signaling. 
We administered a single dose of T3 to P8 mice, and isolated cardiomyocytes from the LV apex or base, either immediately after T3 administration $(0 \mathrm{~h})$ or after $24 \mathrm{~h}$ (Fig. 4A). T3 increased IGF-1 and IGF-1R (Y1131) and MEK1/2 (S218/S222) phosphorylation similarly, but the fraction of total ERK1/2 that was phosphorylated was $~ 50 \%$ lower in cardiomyocytes derived from the LV base than in those of the LV apex (Fig. 4B).

Next, we considered the possibility that reduced ERK1/2 phosphorylation in cardiomyocytes of the LV base might impact its cytosol-to-nucleus translocation. We tested this possibility by repeating the experiment in Fig. 4A, but separating the nuclear and cytosol fractions before assessing total ERK1/2 and phosphorylated ERK1/2 levels. T3-stimulated nuclear translocation of ERK1/2 was similar in cardiomyocytes derived from the LV apex and the LV base (Fig. 4C). However, the fraction of nuclear ERK1/2 that was phosphorylated $24 \mathrm{~h}$ after T3 treatment was $\sim 70 \%$ lower in cardiomyocytes of the LV base than in cardiomyocytes of the LV apex (Fig. 4C).

These data suggest differential dephosphorylation of nuclear p-ERK1/2 between LV cardiomyocytes of the apex and base after it is equivalently translocated from the cytoplasm to the nucleus in both regions. We therefore explored the hypothesis that, after exogenous T3 administration, unequal ratios of nuclear p-ERK1/2-to-total ERK1/2 in these LV cardiomyocytes result from differences in p-ERK1/2-targeting phosphatase(s) in the nucleus.

Dual-specificity phosphatases (DUSPs) are protein tyrosine phosphatases, which dephosphorylate threonine and tyrosine residues on MAPKs. DUSPs are localized to the cytosol, nucleus, or both cell compartments20. Of those that are found in the nucleus (DUSPs 1, 2, 4, 5, 8, 10 and 16) DUSP2, 4 and 5 have high reactivity towards p-ERK1/2. Assessment of DUSP2, 4 and 5 mRNA levels in P8 cardiomyocytes derived from the LV base versus the apex revealed a 0.92-fold $(P=0.85), 0.85$-fold $(P=0.39)$ and 6.3-fold $(P=0.008)$ difference between these LV 
regions, respectively ( $n=4$ /group). Levels of DUSP5 protein were $\sim 6$-fold higher in cardiomyocytes derived from the P8 LV base versus the apex $(P<0.001)$ (Fig. 4C). We therefore explored the potential involvement of DUSP5, which specifically dephosphorylates nuclear p-ERK1/2 21,22, in inhibiting T3-stimulated cardiomyocyte proliferative signaling in cells of the LV base of P8 hearts. In vivo treatment of P8 mice with DUSP5-specific siRNA reduced DUSP5 protein expression in cardiomyocytes of the LV base and, in this setting, unmasked T3mediated stimulation of ERK1/2 phosphorylation and expression of cyclins (Fig. 4D). Collectively, these findings show that DUSP5 inhibits T3-stimulated ERK1/2 phosphorylation in post-P6 cardiomyocytes of the LV base but not apex (Fig. 5).

To determine if the developmental pattern of DUSP5 expression and developmental differences in ERK1/2 activation by exogenous T3 are inversely related, we first examined changes in DUSP5 expression in myocardial tissue derived from the LV apex and base, from birth to P16. DUSP5 was not detectable in the LV apex or base of neonatal animals (Fig. 6A). After P6, DUSP5 expression increased in the LV base and remained high. In the LV apex, DUSP5 expression was first observed at P14; by P15, it was uniformly high throughout the LV. We found that the developmental differences ( $\mathrm{LV}$ apex versus base), at P2, P8 and P16, in exogenous T3-stimulated ERK1/2 phosphorylation and cyclin A2 expression in cardiomyocytes, (Fig. 6B) were inversely related to developmental expression of DUSP5 in the LV myocardium (Fig. 6A).

DUSP5 regulates hyperplastic heart growth in preadolescent mice. We have previously shown that endogenous increases in circulating T3 during the second week of life expand the number of ventricular cardiomyocytes1. Additional studies show developmental increases in ERK1/2 phosphorylation and downstream proliferative signaling in preadolescent LV apical 
cardiomyocytes is also dependent on the T3 surge during this period (Fig. 3D). These studies and the inhibitory effects of DUSP5 expression on exogenous T3-stimulated ERK1/2 phosphorylation in early post-P6 cardiomyocytes (Fig. 4D) led us to hypothesize that ventricular cardiomyocyte proliferation during preadolescence is restrained by DUSP5 expression. We therefore compared the cardiomyocyte endowment and cardiac morphology of 21-day-old Dusp5-/ C57BL/6 mice23 with age-matched wild-type C57BL/6 mice. Dusp5-/- mice had similar body weights to wild-type mice, but their ventricular cardiomyocyte numbers were $16 \%$ higher $(P<0.001)$ (Table 1). In comparison to wild type mice, Dusp5-/ mice had 40\%-60\% thicker LV posterior walls (LVPW), at diastole, at the mid-papillary and mid-apical-levels as well as at the apex $(P<0.001)$ (Table 1). The mid-apical level is mid-distance between the mid-papillary level and the LV apex. Values for the mid-papillary and mid-apical levels were derived from shortaxis measurements, whereas those for the apex were derived from long-axis measurements. Assessments, at diastole, of LV internal dimension (LVID-d) at the mid- papillary level and the mid-apical level indicated that the LV chamber of Dusp5 $-\_$mice was narrower (by $\sim 11 \%$ at both the mid papillary and mid-apical levels; the respective $P$-values were 0.005 and 0.011 ) and longer (LVID-d-long axis) (by 5\%, $P=0.009$ ) (Table 1). In these P21 Dusp5- - hearts, LV fractional shortening was $\sim 8$ points higher $(P<0.001)$ with respect to the short axis and $\sim 5$ points higher with respect to the long axis $(P=0.011)$ (Table 1$)$, and ejection fraction (determined by B-mode echocardiography) was 13 points higher $(P<0.001)$, suggesting better LV contractile function relative to wild-type mice.

The expression of DUSP5 in the early postnatal LV myocardium, starting at the base of the LV in P7 hearts and then proceeding to its apex by P15, together with the early postnatal developmental impact of Dusp5 deletion on total cardiomyocyte numbers, gross LV morphology 
and function, indicates that DUSP5 expression restrains hyperplastic LV growth during early preadolescence.

\section{Discussion}

Here we show that in P8 hearts exogenous T3 stimulates cardiomyocyte proliferation, in vivo, that is restricted to cells in the LV apex. Evidence in support of this conclusion includes studies showing spatial differences in apical versus basal LV cardiomyocytes in T3-stimulated EdU labeling to determine $\mathrm{S}$-phase entry, and in $\mathrm{pH} 3$ labeling to quantify cardiomyocytes in mitosis; increases in cardiomyocyte numbers with T3 treatment, ascertained by careful direct cardiomyocyte counting, and spatial differences in LV apical versus basal cardiomyocyte proliferative responses, as determined by genetic lineage tracing studies. We believe that these multifaceted approaches support our conclusion that exogenous T3 administration can stimulate cardiomyocyte replication in the post-P6 hearts in vivo, albeit that this proliferative response is restricted to cells in the $\mathrm{LV}$ apex.

These studies show that in contrast to cardiomyocytes in the LV base of P6 hearts, those within the apex are not post-mitotic and can respond productively to mitogenic stimulation with T3, which enhances nuclear phospho-ERK1/2 levels and increases cyclin A2 expression. Moreover, as we have previously shown, this proliferative response results in an increase in cardiomyocyte endowment1. We also show that, during postnatal development, resistance to T3proliferative signaling is due to DUSP5 expression, which spreads progressively from LV base at P7 to the apex by P16. Nor is the responsiveness of postnatal LV apical cardiomyocytes merely a pharmacological phenomenon limited to exogenous T3 administration, as it is also observed in response to the naturally occurring surge in endogenous $\mathrm{T} 3$ levels that begins at $\sim \mathrm{P} 10$ in wild 
type C57BL6 mice, resulting in the induction of ERK1/2 phosphorylation and in increased cyclin A2 expression at P13 that are associated with an increase in LV cardiomyocyte numbers between P10 and P16 1. Hence, phenotypic heterogeneity of juvenile cardiomyocytes appears to be an inherent trait of the maturing postnatal heart.

In contrast to our findings, Hirose et al. (2019) 24 propose that endogenous T3 causes cell cycle arrest in postnatal cardiomyocytes. However, their conclusions are based on studies with a genetic mouse model in which a thyroid hormone receptor subtype- $\alpha(\mathrm{TR} \alpha)$ mutation is introduced in cardiomyocytes during embryonic development. In this mutant mouse, in which the affinity of TR $\alpha$ for T3 is reduced, postnatal cell cycle exit is delayed. The structure of TR loci and signaling are complex; strikingly opposing phenotypes could result depending on the TR mutation25, developmental stage and cellular context26. These phenotypes are also highly dependent on the impact of the individual TR mutations on compensatory changes in the expression of other TR subtypes27-30 as well as the consequence of the mutation on the resultant TR $\alpha$ apo-receptor, which regulates extensive gene repression in late fetal heart development. Interpretational complexities associated with this mutant $\operatorname{TR} \alpha$ approach are highlighted by the finding that exogenous T3, given acutely, increases cardiomyocyte proliferation, both in vivo and in vitro, in wild type neonatal mouse hearts 15.

Most laboratories have determined cardiomyocyte numbers by one of two distinct methods: either an indirect histochemical approach in which total cardiomyocyte numbers are extrapolated from the quantitation of a few cells6,31, or by direct cardiomyocyte counting10,24,32. With the first, variable shrinkage of cardiomyocytes can be a confounder due to inconsistent tissue fixation between animals and experiments. A variant of this approach, design-based stereology, requires accurate identification of cardiomyocyte boundaries in situ and involves extrapolations based on 
the evaluation of only a small number of cells sampled randomly from the entire LV. A priori, for these indirect methods to provide valid results requires homogeneity of cardiomyocytes within the heart. It is, thus, not congruent with studies of the developing heart, that, as we show here, is heterogeneous with respect to the replicative capacity of its constituent cardiomyocytes. Direct counting, of cardiomyocytes, on the other hand, is used more extensively10,24,32, but also has its own challenges due to variations in the efficiency of disaggregation of hearts between animal groups and between laboratories. Here, we have optimized this method to overcome this problem by using the same lot of disaggregating enzyme between animal groups and experiments, as well as the same operator to quantitate cardiomyocyte numbers. Furthermore, we mildly fix the hearts before enzymatic disaggregation so that over-digestion does not yield cardiomyocyte fragments that could be mistakenly assumed to be heart cells15. Such optimization of our disaggregation technique allows digestion of entire hearts with greater than $97 \%$ efficiency, which is maintained both between experiments and with different groups of animals.

Importantly, the findings presented here are not only of biological interest, but may well explain the inability of some others 12 to confirm our findings of a T3-driven cardiomyocyte proliferative burst during the second week of life 1 . This because developmentally-regulated spatial differences in cardiomyocyte proliferation were not considered in these other studies13, making the detection of proliferation in only a subset of cardiomyocytes problematic.

Our studies indicate that developmentally regulated expression of DUSP5, a nuclear ERK1/2-specific phosphatase, which begins in the LV base and spreads progressively to cardiomyocytes of the LV apex, is the molecular signal that triggers resistance to the T3 mitogenic response. Thus, acute DUSP5 depletion reverses the refractoriness of cardiomyocytes in the LV base of P8 hearts to T3, allowing these cells to now display a T3-mediated response: 
enhanced nuclear phospho-ERK1/2 levels and cyclin activation.

Detailed changes in LV morphology, resulting from significant levels of cardiomyocyte hyperplasia, have been reported infrequently. Using transthoracic echocardiography, we show that Dusp5-deletion-induced cardiomyocyte hyperplasia is associated with an altered cardiac morphology as a result of an increase in LV mass and LV wall thickness that narrows LV chamber dimensions and improve LV contractile function, as evident from increases in LV fractional shortening and LV ejection fraction. Importantly, in contrast to other genetic models of enhanced cardiomyocyte proliferative growth 31,32 , the morphological changes observed here during development in Dusp5-^- mice do not impact negatively on stroke volume, bodyweight or survival.

In summary, our studies provide evidence for inhomogeneity of LV cardiomyocytes during early postnatal heart development that underlies spatial differences in mitogenic responsiveness. Exploiting mechanism described here may prove useful in expanding the number of cardiomyocytes in adults.

\section{Materials and Methods}

A detailed description of the experimental procedures related to cardiomyocyte isolation for immunocytochemistry, RT-qPCR and immunoblotting is provided in the Supplementary Information section.

\section{Animal husbandry and mouse models}

All animal studies were approved by the Institutional Animal Care and Use Committee (IACUC) of Emory University. We confirm that all experiments were performed in accordance with IACUC guidelines and regulations. Adult C57BL/6 mice were used for breeding. Conditions for 
animal husbandry protocol are adapted from our earlier studies15. Briefly, the mothers were between 3-8-months-old. Mice were allowed food and water ad libitum. An animal husbandry protocol was developed to minimize variation between litters and between studies. Factors considered were as follows: 1) age of the mother (only dams less than 36 weeks were used); 2) animal chow (we used a 50/50 mix of Purina Lab Diets Rodent diet 5001 (standard diet) and 5015 (breeder diet); 3) minimization of stress on the dams, especially between the first 2 days after delivery and weaning; 4) standardization of litter sizes (only pups from litter sizes of 6-8 (at P2) were used); 5) litter sizes from these births were further adjusted to 4 pups per dam at P2 to minimize inter-experiment variation in growth; 6) to minimize intra-litter variation, drugs were given to about one half of the pups in a litter and vehicle to the rest; and 7) separation of plugged/pregnant dams into individual cages. C57BL/6 wild type (Jackson Laboratory, 000664) male and female mice were used for these studies. Wherever possible we constituted each adjusted litter with equal numbers of male and female mice.

For cardiomyocyte lineage tracing studies, we used Rosa26fs-Confetti [B6.129P2Gt(ROSA)26Sortm1(CAG-Brainbow2.1)Cle/J, Jackson Laboratory, 017492] and Myh6MerCreMer [B6.FVB(129)-A1cfTg(Myh6-cre/Esr1*)1Jmk/J, Jackson Laboratory, 005657] mice. We then generated double-transgenic Myh6-MerCreMer::Rosa26fs-Confetti mice by breeding. In the double transgenic mice, Cre recombinase causes the Brainbow 2.1 construct to recombine, which randomly labels cardiomyocytes either with GFP, CFP, RFP or YFP. We limited the extent of Cre-mediated recombination by adjusting the dose of 4-hydroxytamoxifen (Sigma, H7904-5mg) to minimize replication-independent occurrences of adjacent cardiomyocytes of the same color. For studying the biological role of DUSP5 in postnatal heart development we used Dusp5-/ mice on the C57BL/6 background23. 
Drugs such as T3 and 4-hydroxytamoxifen were administered, intraperitoneally (i.p.), to each mouse at the dose indicated. Propylthiouracil (PTU, 0.15\%) was administered in drinking water to inhibit T3 biosynthesis in mice from P7 to P13 using the protocol from our earlier studies1. Vehicle was administered by the same route and animals thus treated served as controls. Phosphate buffered saline (PBS) was the vehicle for T3 and soybean oil was the vehicle for 4hydroxytamoxifen. DUSP5-specific siRNA or scrambled siRNA (control) was administered using in vivo-jetPEI ${ }^{\circledR},(\mathrm{VWR}, 89129-960)$. DUSP5 siRNA (100 $\left.\mu \mathrm{g}\right)$ was dissolved in $1 \mathrm{ml}$ of the in vivo-jetPEI:10\% glucose mixture and was injected $100 \mu \mathrm{l}$ per mouse via i.p. route (10 $\mu \mathrm{g} /$ mouse). DUSP5 siRNA (sc-60555) was a pool of 2 different siRNA duplexes (sc-60555A, sense: CAUGGCUUACCUCAUGAAtt and antisense: UUCAUGAGGUAAGCCAUGCtt; sc60555B, sense: GACAGCUCCUUCAGUAUGAtt and antisense: UCAUACUGAAGGAGCUGUCtt). All sequences are provided in $5^{\prime} \rightarrow 3^{\prime}$ orientation. We did not use any litters if one or more mouse in the litter was observed to be sick or stunted. P7 and older mice were first anesthetized with 5\% isoflurane and then hearts were harvested for further processing. Hearts were also collected at 0,24 and $40 \mathrm{~h}$ after $\mathrm{T} 3$ or vehicle treatment and ventricular cardiomyocytes were prepared from the apex and base for immunoblotting, RT-qPCR or immunocytochemistry. In addition, hearts were collected at various ages after birth for immunoblotting, immunocytochemistry, immunohistochemistry and cardiomyocyte number estimation.

\section{Echocardiography analysis}

Transthoracic echocardiography was performed under light isoflurane anesthesia on either wildtype controls or Dusp5-/- mice at P21 using the Vevo 3100 imaging platform (VisualSonics Inc.) with a MX550D linear array transducer (axial resolution: $40 \mu \mathrm{m}$ ) and the images were 
analyzed using Vevo® Lab Desktop Software. The automated zoom function of the Vevo 3100 imaging platform allows precise quantification. Both B-mode and M-mode measurements were taken to assess the cardiac morphology and function. Parasternal short axis M-mode echocardiographic imaging was used to determine wall thicknesses and internal diameter of the LV at diastole and systole; fractional shortening was derived from these parameters. IVS function of M-mode (minor axis) of Vevo® Lab Desktop Software was used to determine these parameters. Endocardium and epicardium were outlined using the IVS function by sequential click-defining the anterior epicardial, endocardial and then posterior endocardial and epicardial wall. These measurements were made at the mid-apical level (half way between the apex and the LV mid papillary level; $\sim 1 \mathrm{~mm}$ from the apex) or mid-papillary level ( $\sim 3.5 \mathrm{~mm}$ from the apex). Additionally, B-mode parasternal long axis images were acquired to determine LV major axis internal diameters and wall thicknesses. Using B-mode (major axis), length of the LV chamber was determined by measuring distance from the aortic root to the apical endocardial wall at systole and diastole. We also measured LVPW thickness at the apex using B-mode (long-axis) by sequential click-defining apical wall from the epicardium to endocardium. LV volumes at diastole and systole were also determined using B-mode parasternal long axis images of the heart. LV ejection fraction measurements were calculated using the LV-trace function of the Vevo ${ }^{\circledR}$ Lab Desktop Software in B-mode. LV endocardial borders were traced at end-diastole and end-systole by sequential click-defining the aortic root, apex, anterior wall, posterior wall and repeat click-defining of anterior and posterior wall until the myocardial wall is outlined. The Vevo ${ }^{\circledR}$ Lab Desktop Software automatically determines volumes at end-diastole, end-systole, and calculates LV volume, SV and LV ejection fraction. At least three different images were taken for each cardiac parameter and measurements from the these three individual images were 
averaged to acquire final measurement for that cardiac parameter.

\section{Cardiomyocyte number determination}

Protocol for cardiomyocyte number determination is customized based on our earlier studies 15. Briefly, heparin (100--200 $\mu$ l, 1000 USP units/ml) was injected intraperitoneally 8 minutes prior to harvesting. Hearts were harvested under deep anesthesia using 5\% isoflurane. Hearts with their atria and aorta attached were washed with PBS and then the aorta cannulated for retrograde perfusion through the coronary circulation. Hearts were immediately perfused with cytofix (BD Biosciences, 554655) for $1 \mathrm{~min}$. Subsequently, hearts were perfused with perfusion buffer (120 $\mathrm{mmol} / \mathrm{L} \mathrm{NaCl}, 15 \mathrm{mmol} / \mathrm{L} \mathrm{KCl}, 0.5 \mathrm{mmol} / \mathrm{L} \mathrm{KH}_{2} \mathrm{PO}_{4}, 5 \mathrm{mmol} / \mathrm{L} \mathrm{NaHCO} 3,10 \mathrm{mmol} / \mathrm{L}$ HEPES, and $5 \mathrm{mmol} / \mathrm{L}$ glucose, at $\mathrm{pH}$ 7.0) for $2 \mathrm{~min}$ and then with perfusion buffer containing collagenase type 2 (Worthington, LS004176) for $8-15$ min at $37^{\circ} \mathrm{C}$. Perfusion and digestion buffers were freshly prepared, warmed to $37{ }^{\circ} \mathrm{C}$ and aerated with $5 \% \mathrm{CO}_{2}$. Collagenase concentration was $1 \mathrm{mg} / \mathrm{ml}$ for P8 to P12 hearts and $2 \mathrm{mg} / \mathrm{ml}$ for P16 or older hearts. After 8-15 min of digestion, the atria were excised and the cardiac ventricles were placed in a $6 \mathrm{~cm}$ dish containing $2 \mathrm{ml}$ of digestion buffer; we then added $\sim 2 \mathrm{ml}$ of STOP buffer (perfusion buffer plus $10 \%$ bovine calf serum and $12.5 \mathrm{mmol} / \mathrm{L} \mathrm{CaCl}_{2}$ ). The ventricles were teased apart into small pieces followed by trituration through pipettes of progressively smaller diameters. The digested cardiomyocytes from each heart were collected in a $15 \mathrm{ml}$ falcon tube and more STOP buffer was added to a volume of $10 \mathrm{ml}$. The final cell suspension was used to count cardiomyocytes using a hemocytometer. To avoid losses, cardiomyocytes were not purified, but could be readily identified by phase contrast microscopy based on their cytoplasmic size and rod shape. Four aliquots were counted per heart and the mean value was used to determine the total number of ventricular cardiomyocytes per heart. 
For accurate cardiomyocyte number determination, a critical step is optimal digestion efficiency and operator-specific variability in isolation and counting. We have eliminated operator-specific variability by using the same operator between experiments. Heart digestion is chiefly dependent on collagenase concentration in the perfusion medium, its activity, exposure time and temperature. To optimize digestion efficiencies, these variables were adjusted for each group of mice depending on the age of the mouse. Importantly, collagenase activity was kept uniform between the biological replicates and across experiments by using the same lot of enzyme. Additionally, a brief fixation with cytofix ( $1 \mathrm{~min})$ before starting perfusion with collagenase helps in protecting individual cardiomyocyte structure and prevents generation of fragmented cardiomyocytes. Cardiomyocytes during the postnatal stages analyzed are much larger than non-myocytes and are readily identifiable due to their cytoplasmic size and rodshape15. Digestion efficiency was calculated [ventricular weight $\%$, determined by (original weight - residual)/original weight] after each change in condition. We found that maximal digestion efficiencies were between $\sim 97 \%$ and $99 \%$. Upon microscopic examination, the residual tissue was almost entirely undigested cardiac valves and blood vessels. Over-digestion neither improved digestion efficiencies, nor did it increase cardiomyocyte yield. We did not estimate cardiomyocyte numbers from under-digested hearts in which disaggregation of myocardial tissue was incomplete. Suboptimal cannulation of the aorta was the cause of underdigestion but was infrequent.

\section{Immunofluorescence}

Cardiomyocytes were isolated as described above and fixed in Cytofix (BD Biosciences, 554655) for $5 \mathrm{~min}$. After pre-blocking, cardiomyocytes were stained with anti-cardiac troponin T- (Miltenyi Biotec, 130-119-674), or anti-phospho-histone H3-AlexaFluor 594 conjugate (Cell 
signaling, $8481 \mathrm{~S}$ ) in $10 \% \mathrm{v} / \mathrm{v}$ goat serum. EdU positive cardiomyocyte nuclei were detected using Click-iT EdU Alexa Fluor 594 Imaging Kit (Invirogen, C10339). DAPI was used to stain cardiomyocyte nuclei. Images were acquired on a confocal microscope (Leica SP5).

\section{Statistical analysis}

Statistical significance of data was determined using Graphpad Prism 8. The Shapiro-Wilk test was used to determine if the data were normally distributed; in this case, we used one-way ANOVA followed by Tukey multiple comparisons test, or unpaired two-tailed Student's $t$-test for comparisons involving 2 groups, or paired $t$-test for intra-cardiac comparison of the apex with the base of the same heart. For estimation of variance, the F-test was used when comparing 2 groups and the Brown-Forsythe test was used when comparing multiple groups by 1-way ANOVA. $P$-values $<0.05$ were considered significant. Results are expressed as mean $\pm \mathrm{SEM}$ or as Tukey's box and whisker plots.

\section{Data availability}

The data and resources generated for this manuscript are available upon reasonable request from the corresponding authors.

\section{References}

1. Naqvi, N. et al. A proliferative burst during preadolescence establishes the final cardiomyocyte number. Cell 157, 795-807 (2014).

2. Krenz, M. \& Robbins, J. Impact of beta-myosin heavy chain expression on cardiac function during stress. J. Am. Coll. Cardiol. 44, 2390-2397 (2004).

3. Soonpaa, M. H., Kim, K. K., Pajak, L., Franklin, M. \& Field, L. J. Cardiomyocyte DNA synthesis and binucleation during murine development. Am. J. Physiol. 271, H2183-H2189 
(1996).

4. Bai, S. L., Campbell, S. E., Moore, J. A., Morales, M. C. \& Gerdes, A. M. Influence of age, growth, and sex on cardiac myocyte size and number in rats. Anat. Rec. 226, 207-212 (1990).

5. Li, F., Wang, X., Capasso, J. M. \& Gerdes, A. M. Rapid transition of cardiac myocytes from hyperplasia to hypertrophy during postnatal development. J. Mol. Cell Cardiol. 28, 1737$1746(1996)$.

6. Alkass, K. et al. No evidence for cardiomyocyte number expansion in preadolescent mice. Cell 163, 1026-1036 (2015).

7. Naqvi, N. et al. Cardiomyocytes replicate and their numbers increase in young hearts. Cell 163, 783-784, (2015).

8. Zhang, C. H. \& Kühn, B. Muscling up the heart: a preadolescent cardiomyocyte proliferation contributes to heart growth. Circ. Res. 115, 690-692 (2014).

9. Foglia, M. J. \& Poss, K. D. Building and re-building the heart by cardiomyocyte proliferation. Development 143, 729-740 (2016).

10. Puente, B.N. et al. The oxygen-rich postnatal environment induces cardiomyocyte cell-cycle arrest through DNA damage response. Cell 157, 565-579 (2014).

11. Mollova, M. et al. Cardiomyocyte proliferation contributes to heart growth in young humans. Proc. Natl. Acad. Sci. U S A. 110, 1446-1451 (2013).

12. Hirai M. et al. Tissue-specific cell cycle indicator reveals unexpected findings for cardiac myocyte proliferation. Circ Res 118, 20-28 (2016).

13. Soonpaa, M.H. et al. Cardiomyocyte cell cycle activity during preadolescence. Cell 163, 751752 (2015). 
14. Bergmann, O. et al. Dynamics of cell generation and turnover in the human heart. Cell 161, 1566-1575 (2015).

15. Tan, L. et al. Redox activation of JNK $2 \alpha 2$ mediates thyroid hormone-stimulated proliferation of neonatal murine cardiomyocytes. Sci. Rep. 9, 17731 (2019).

16. Snippert, H. J. et al. Intestinal crypt homeostasis results from neutral competition between symmetrically dividing Lgr5 stem cells. Cell 143, 134-144 (2010).

17. Chang, L. \& Karin, M. Mammalian MAP kinase signalling cascades. Nature 410, 37-40 (2001).

18. Mebratu, Y. \& Tesfaigzi, Y. How ERK1/2 activation controls cell proliferation and cell death: Is subcellular localization the answer? Cell Cycle 8, 1168-1175 (2009).

19. Oberkotter, L. V. \& Rasmãoessen, K. M. Changes in plasma thyroid hormone concentrations in chronically food-restricted female rats and their offspring during suckling. J. Nutr. 122, 435-441 (1991).

20. Jeffrey, K. L., Camps, M., Rommel, C. \& Mackay, C. R. Targeting dual-specificity phosphatases: manipulating MAP kinase signalling and immune responses. Nat. Rev. Drug Discov. 6, 391-403 (2007)

21. Keyse, S. M. Dual-specificity MAP kinase phosphatases (MKPs) and cancer. Cancer Metastasis Rev. 27, 253-261 (2008).

22. Caunt, C.J. \& Keyse, S. M. Dual-specificity MAP kinase phosphatases (MKPs): shaping the outcome of MAP kinase signalling. FEBS J. 280, 489-504 (2013).

23. Kutty, R. G. et al. Dual specificity phosphatase 5 is essential for T cell survival. PLoS One 11, e0167246 (2016).

24. Hirose, K. et al. Evidence for hormonal control of heart regenerative capacity during 
endothermy acquisition. Science 364, 184-188 (2019).

25. Flamant, F \& Samarut, J. Thyroid hormone receptors: Lessons from knockout and knock-in mutant mice. Trends Endocrinol. Metab. 14, 85-90 (2003).

26. Puzianowska-Kuznicka, M., Pietrzak, M., Turowska, O. \& Nauman, A. Thyroid hormones and their receptors in the regulation of cell proliferation. Acta Biochim. Pol. 53, 641-650 (2006).

27. Chatonnet, F., Guyot, R., Benoit, G. \& Flamant, F. Genome-wide analysis of thyroid hormone receptors shared and specific functions in neural cells. Proc. Natl. Acad. Sci. USA. 110, E766-E775 (2013).

28. Ortiga-Carvalho, T. M., Sidhaye, A. R. \& Wondisford, F. E. Thyroid hormone receptors and resistance to thyroid hormone disorders. Nat. Rev. Endocrinol. 10, 582-591 (2014).

29. Kaneshige, M. et al. A targeted dominant negative mutation of the thyroid hormone $\alpha 1$ receptor causes increased mortality, infertility, and dwarfism in mice. Proc. Natl. Acad. Sci. USA. 98, 15095-15100 (2001).

30. Forrest, D. \& Vennström, B. Functions of thyroid hormone receptors in mice. Thyroid 10, 41-52 (2000).

31. Chaudhry, H. W. et al. Cyclin A2 mediates cardiomyocyte mitosis in the postmitotic myocardium. J. Biol. Chem. 279, 35858-35866 (2004).

32. D'Uva, G. et al. ERBB2 triggers mammalian heart regeneration by promoting cardiomyocyte dedifferentiation and proliferation. Nat. Cell Biol. 17, 627-638 (2015).

\section{Acknowledgments}

This work was supported by grants from the Department of Medicine, Emory University, the Carlyle Fraser Heart Center, Emory University Hospital Midtown, the NIH (HL079040, 
HL127726, HL098481, T32HL007745, HL092141, HL093579, HL094373, HL113452,

HL123338 and T32HL007745), the American Heart Association (13SDG16460006;

17GRNT33670975), the Fondation Leducq Transatlantic Network, National Health and Medical

Research Council, Australia (APP1074386), R.T. Hall estate, the Australian Research Council

Stem Cells Australia, Special Initiative in Stem Cell Science grant (SR1101002) and the

Department of Pediatrics, Children's Research Institute, Medical College of Wisconsin.

\section{Author Contributions}

N.N, and A.H. were responsible for the original concept and design of primary experiments.

N.B. performed echocardiography to acquire images for L.T. to analyze, determined

cardiomyocyte number and also isolated cardiomyocytes for western blotting. L.T. performed western blotting, drugs administration and tissue harvesting and analysis of echocardiography images. H.N. and E. F. performed quantitative RT-qPCR. J.W.C. assisted N.N. in performing immunohistochemical studies. A.G. and R.R. developed and characterized Dusp5-- mice. A.H., N.N., R.M.G., S.E.I., and D.I.K.M. prepared the manuscript. All authors discussed the results and edited the manuscript.

\section{Additional Information}

Supplementary information includes methods and one Supplementary Table. A separate pdf file with all the uncut immunoblotting gels is also included. Some antibodies generate background and for these reasons we have additionally provided over and under-exposed images of gels for these protein targets.

Competing Interests: The authors declare no competing interests. 


\section{Figures with Legends}
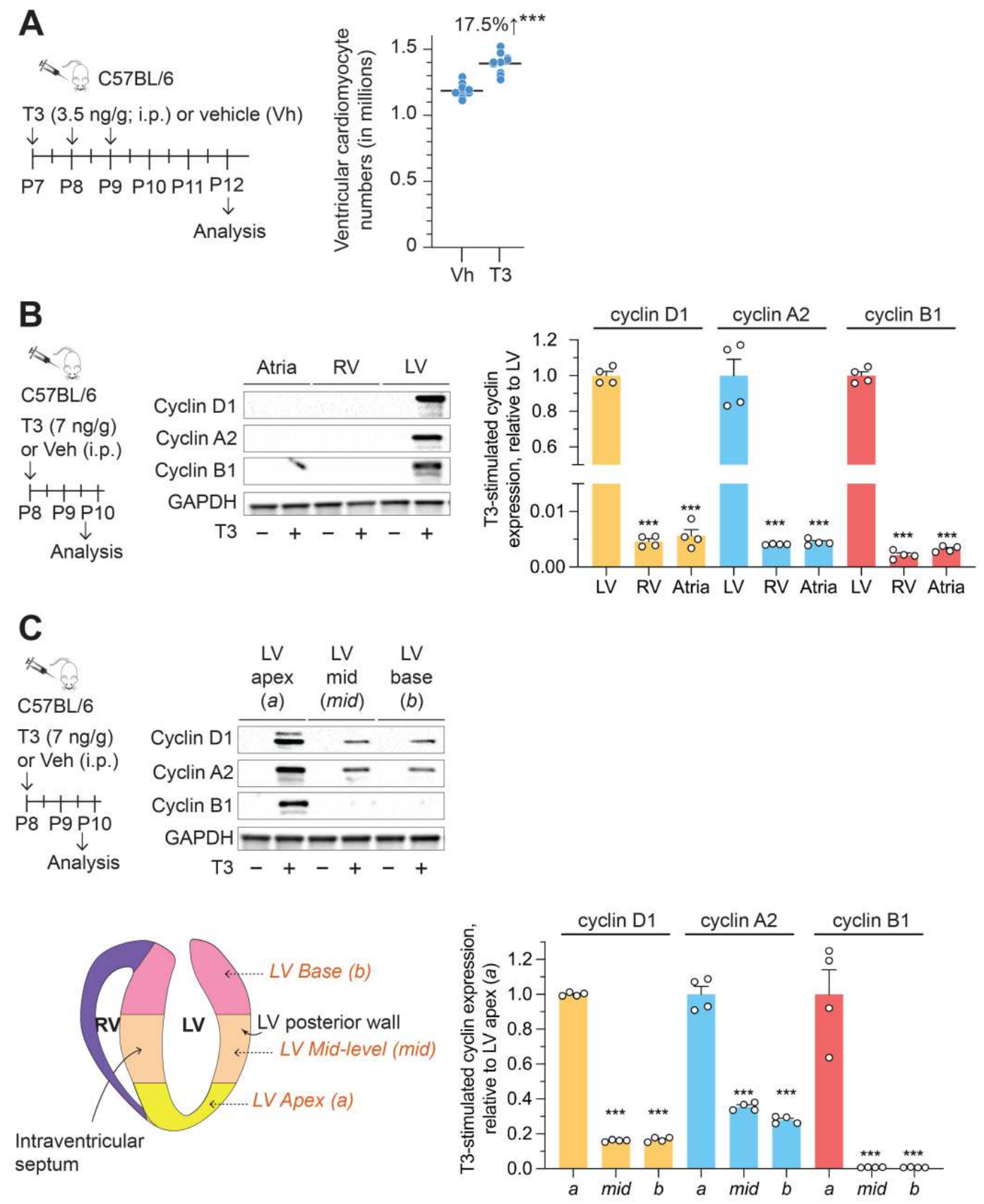

Figure 1. T3 increases ventricular cardiomyocyte proliferation and cyclin expression in the LV myocardium of post-P6 hearts. (A) Exogenous T3 administration increases ventricular cardiomyocyte numbers in $\mathrm{P} 7$ mice. $* * * P<0.001$. Schematics illustrate experimental protocol. $(\mathbf{B}, \mathbf{C})$ In vivo $\mathrm{T} 3$ administration increases cyclin $\mathrm{D} 1, \mathrm{~A} 2$, and $\mathrm{B} 1$ expression in the $\mathrm{LV}$, but not atria or right ventricle $(\mathrm{RV})(B)$ and, within the $\mathrm{LV}$, preferentially in the apex compared to the mid LV or base $(C)$. Histograms show the quantitation of the data in $(B)$ and $(C)$. Immunoblots in $\mathrm{B}$ and $\mathrm{C}$ are representative of 4 biologically independent replicates. Schematics illustrate experimental protocol. Error bars indicate SEM. $* * * P<0.001$. 
A

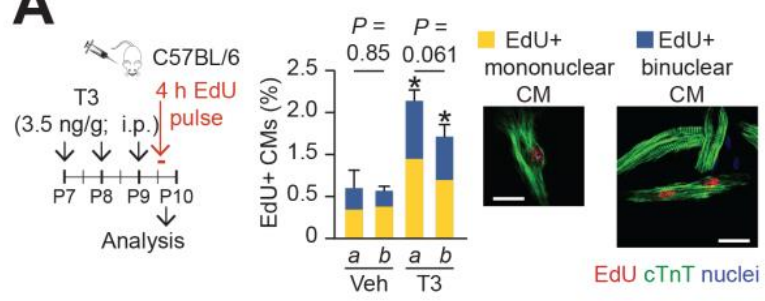

C

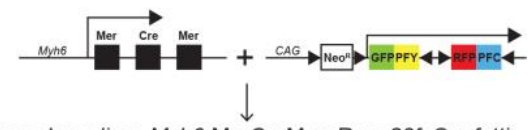

Cross breeding: Myh6-MerCreMer::Rosa26fsConfetti double transgenic 4-OH-tamoxifen pulse
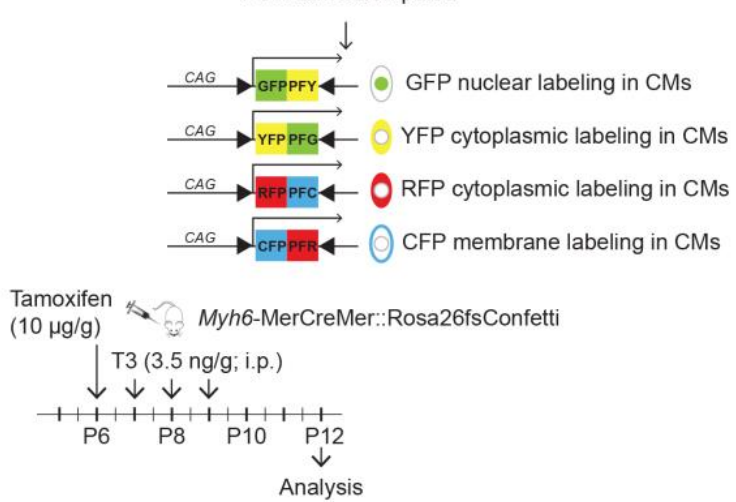

B
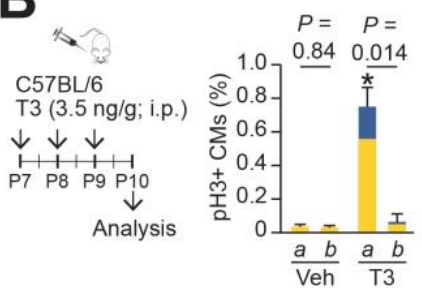

$\underset{\text { mononuclear }}{\mathrm{pH} 3+} \underset{\text { binuclear }}{\mathrm{pH} 3+}$

$\mathrm{CM} \quad \mathrm{CM}$

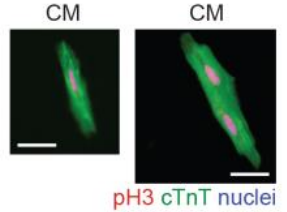

LV base; total LV apex; total

$\mathrm{CM}$ clusters $=8 \mathrm{CM}$ clusters $=41 \square 2-\mathrm{CM}$ cluster

$\square$ 3-CM cluster

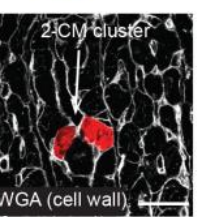

- 4-CM cluster
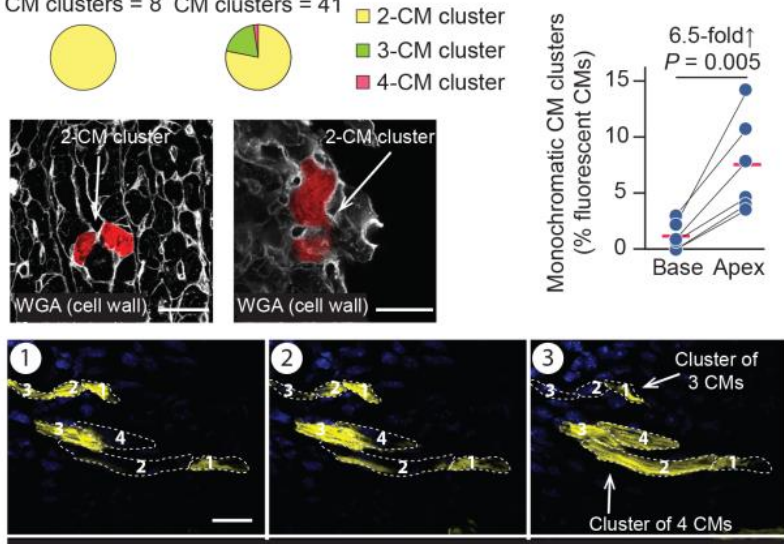

Sequential optical planes (1-3) of a high power field using confocal microscopy

Figure 2. LV apical cardiomyocytes (CM) of post-P6 hearts proliferate in response to exogenous T3 versus vehicle. (A, B) Effects of T3 on DNA synthesis (EdU labeling) (A) and mitosis (pH3 labeling) (B) in cardiomyocytes from LV apex $(a)$ or base $(b)$. Cardiomyocytes were identified by cTnT labeling. $* P<0.05$ for vehicle (Veh) versus T3 comparisons within each region using an unpaired Student's $t$-test; $n=4$. Intra-LV comparisons were made using a paired Student's $t$ test; $P$-values for these comparisons are indicated. Between 500 and 2,000 cardiomyocytes were analyzed for each assessment. (C) Multicolor labeling of post-neonatal mouse LV cardiomyocytes. Limited 4-hydroxytamoxifen-induced recombination of paired loxP sites (black triangles) leads to expression of green fluorescent protein (GFP), yellow fluorescent protein (YFP), red fluorescent protein (RFP) or cyan fluorescent protein (CFP) in a few cardiomyocytes, randomly positioned within the entire LV. Insets show examples of monochromatic clusters. Intra-LV comparisons were made using a paired Students $t$-test. Schematics illustrate experimental protocol. Error bars indicate SEM. White bars in photomicrographs represent 20 $\mu \mathrm{m}$. 

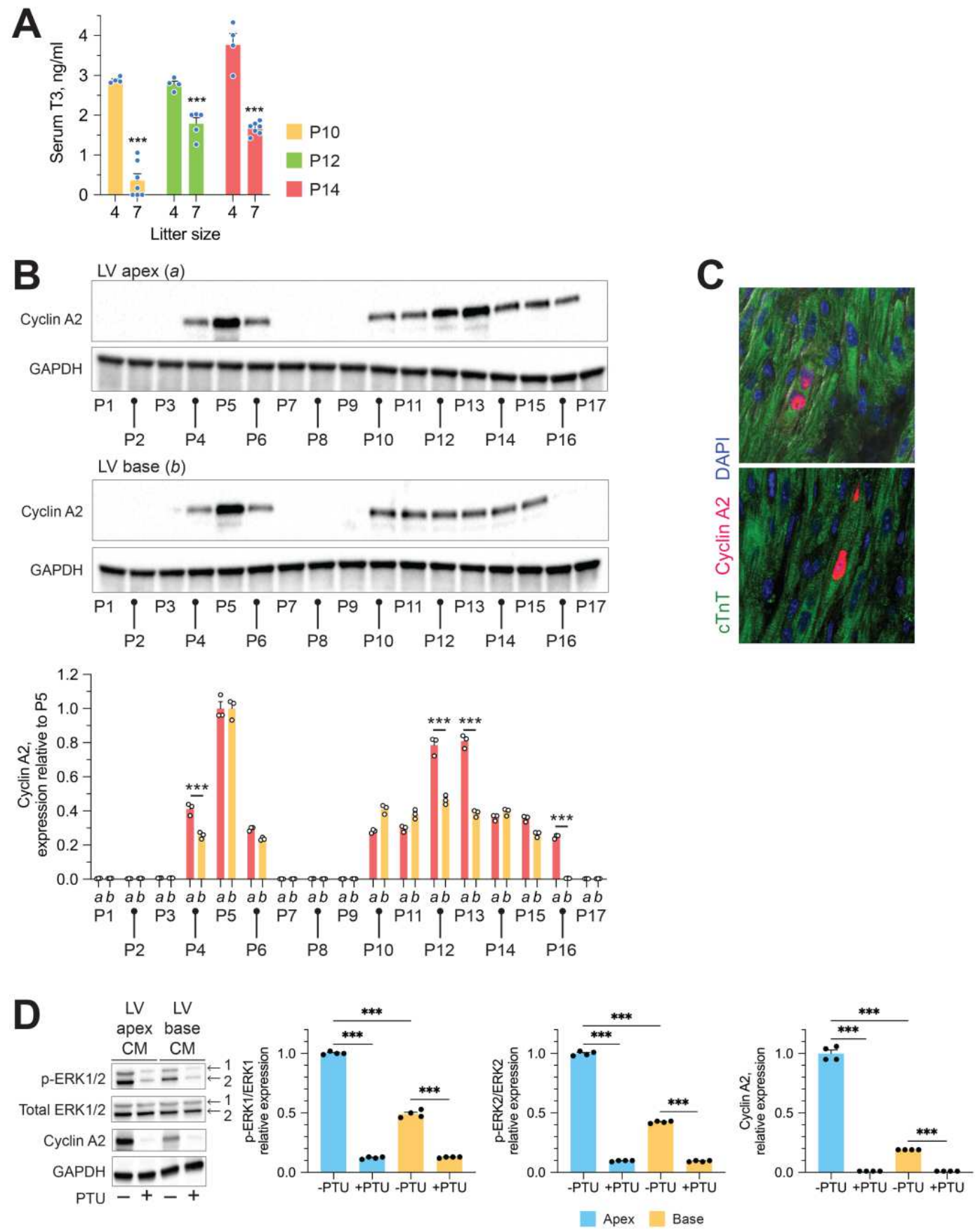

Figure 3. Spatial restriction of cardiomyocytes proliferative signaling to the LV apex during preadolescence. (A) Effect of litter size on developmental surge in circulating T3 during early preadolescence. (B) Representative immunoblots showing developmental changes in myocardial Cyclin A2 levels in the LV apex $(a)$ and base $(b)$ of early postnatal hearts. Bar graphs show GAPDH-normalized cyclin A2 levels relative to levels in P5 LV myocardium. (C) Two examples of a cyclin A2+ cardiomyocyte in a P13 LV apical tissue section. No cyclin A2+ cardiomyocytes were seen on sections of P13 LV base. (D) Representative immunoblots show expression levels of indicated proteins in whole cardiomyocyte (CM) lysates obtained from CMs 
of the LV apex and base at P13. Immunoblots show that developmental increases in p-ERK1/2 and Cyclin A2 in LV apical CMs of P13 mice are suppressed by PTU treatment that blocks T3 biosynthesis. Quantitative data is shown next to the immunoblots. ${ }^{*} * * P<0.001$. Data are mean \pm SEM. 
A

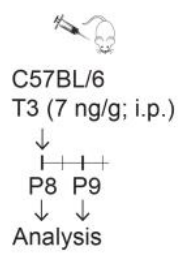

CM cytosolic CM nuclear

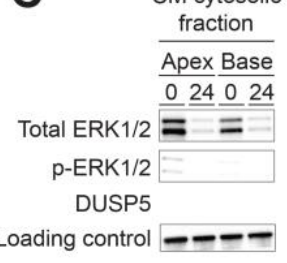

D

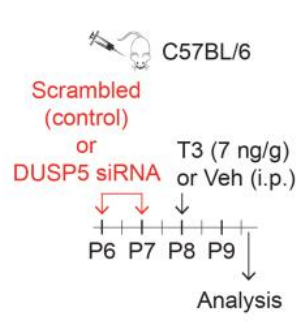

Loading control

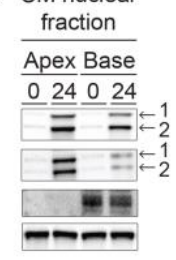

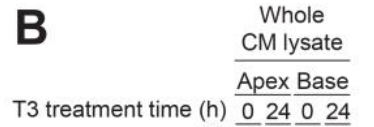

T3 treatment time (h) $0 \underline{24} \underline{0} \underline{24}$

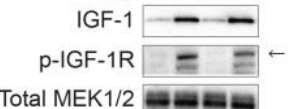

Total MEK1/2 $\mathbf{m}=\mathbf{a}$

p-MEK1/2

Total ERK1/2 $\mathbb{E}=\mathbb{E} \leftleftarrows 2$

$\mathrm{p}-\mathrm{ERK} 1 / 2 \square= \pm \leftarrow 1$

GAPDH $\longrightarrow$
T3-stimulated proteins,
relative expression

relative expression

Apex Base

IGF-1 $1.0 \pm 0.01 \quad 1.01 \pm 0.02$

p-IGF-1R $\quad 1.0 \pm 0.03 \quad 1.01 \pm 0.02$

p-MEK/MEK $\quad 1.0 \pm 0.03 \quad 1.02 \pm 0.05$

p-ERK1/ERK1 $1.0 \pm 0.020 .46 \pm 0.01^{* * *}$

p-ERK2/ERK2 $1.0 \pm 0.02 \quad 0.69 \pm 0.01^{\text {*** }}$

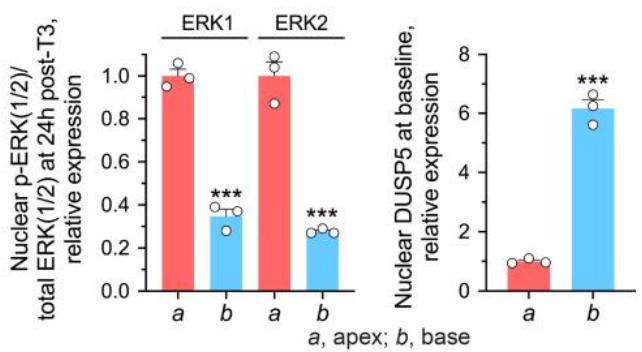

LV apex (a) CMs LV base (b) CMs
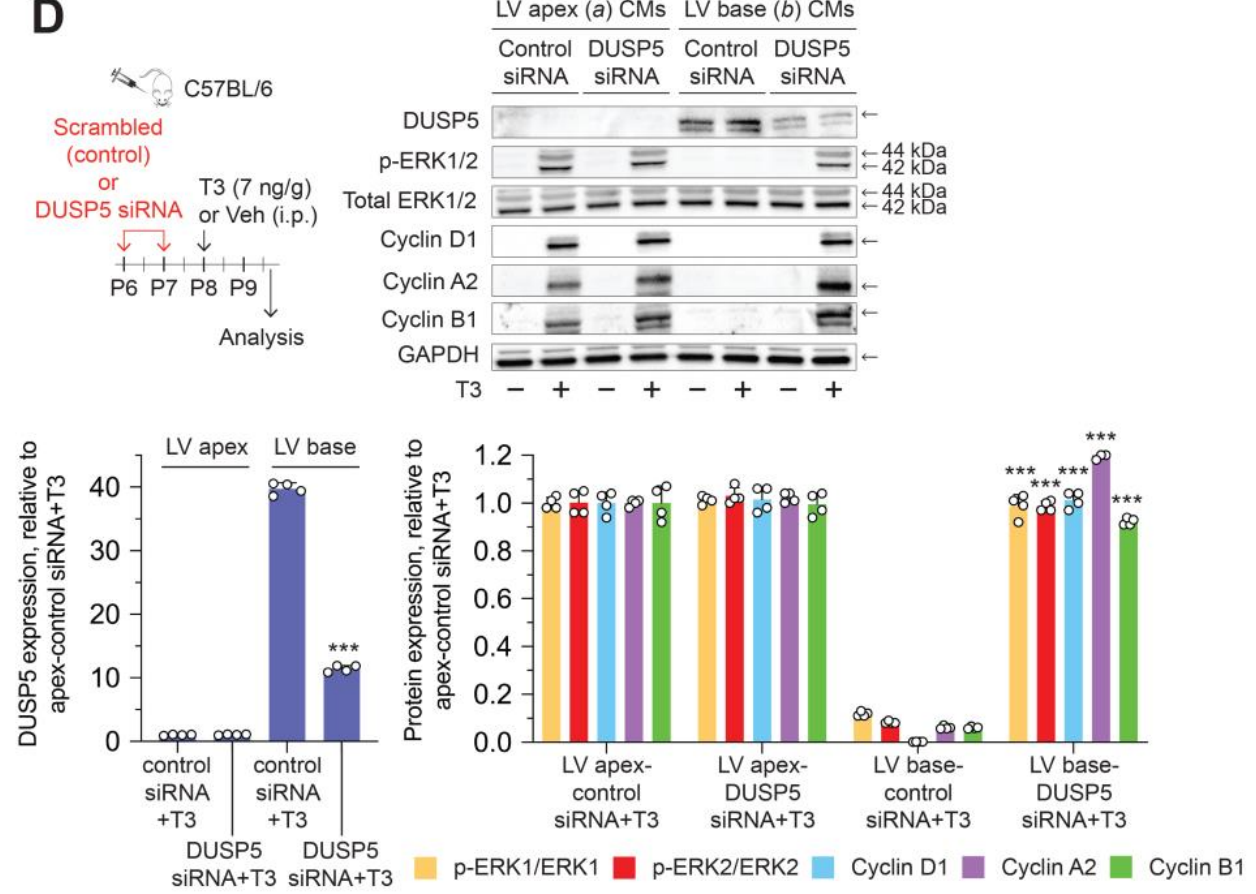

Figure 4. T3-induced ERK1/2 phosphorylation and cyclin expression in cardiomyocytes (CMs) of the P8 LV base is inhibited by DUSP5. (A) Schematic showing the protocol for T3 administration and analysis times. (B) Representative immunoblots show expression levels of indicated proteins in whole CM lysates obtained from CMs of the LV apex and base at 0 (baseline) and $24 \mathrm{~h}$ after T3 administration. Quantitative data relative to that obtained from analysis of CMs of the LV apex, are shown next to the immunoblots. (C) Using the same protocol as in $\mathrm{A}$, isolated $\mathrm{CMs}$ were resolved into their constituent nuclear and cytosol fractions 
before immunoblot analysis. Quantitative data are shown in bar graphs adjacent to the immunoblots. In B and C, immunoblots are representative of 4 biologically independent replicates. (D) DUSP5 or scrambled (control) siRNA was administered intraperitoneally (i.p.) to mice at P6 and P7, followed by T3 (+) or vehicle (-) treatment at P8. siRNAs were dissolved in the in vivo-jetPEI and $10 \%$ glucose mixture. Forty hours later, CMs were isolated and whole cell lysates prepared. Lysates were then subjected to immunoblotting for the indicated proteins. Immunoblots are representative of 4 biologically independent replicates. Quantitative data are presented in the histograms. Data are mean \pm SEM. $* * * P<0.001$ comparisons for intra-LV region derived cardiomyocytes from control versus DUSP5 siRNA treated mice. 


\section{Cardiomyocytes of the P7-P8 LV base}

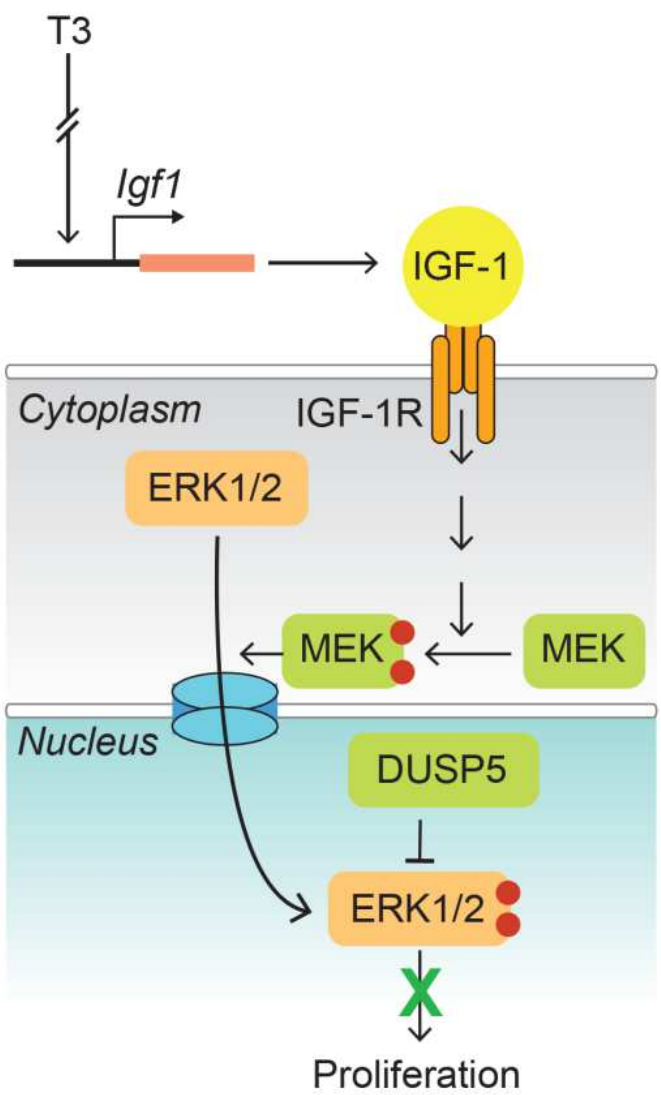

- Phosphorylation

Figure 5. A working model illustrating the mechanism underlying the selective loss of nuclear phosphorylated ERK1/2 in cardiomyocytes from the base of the LV of post-P6 mice. 

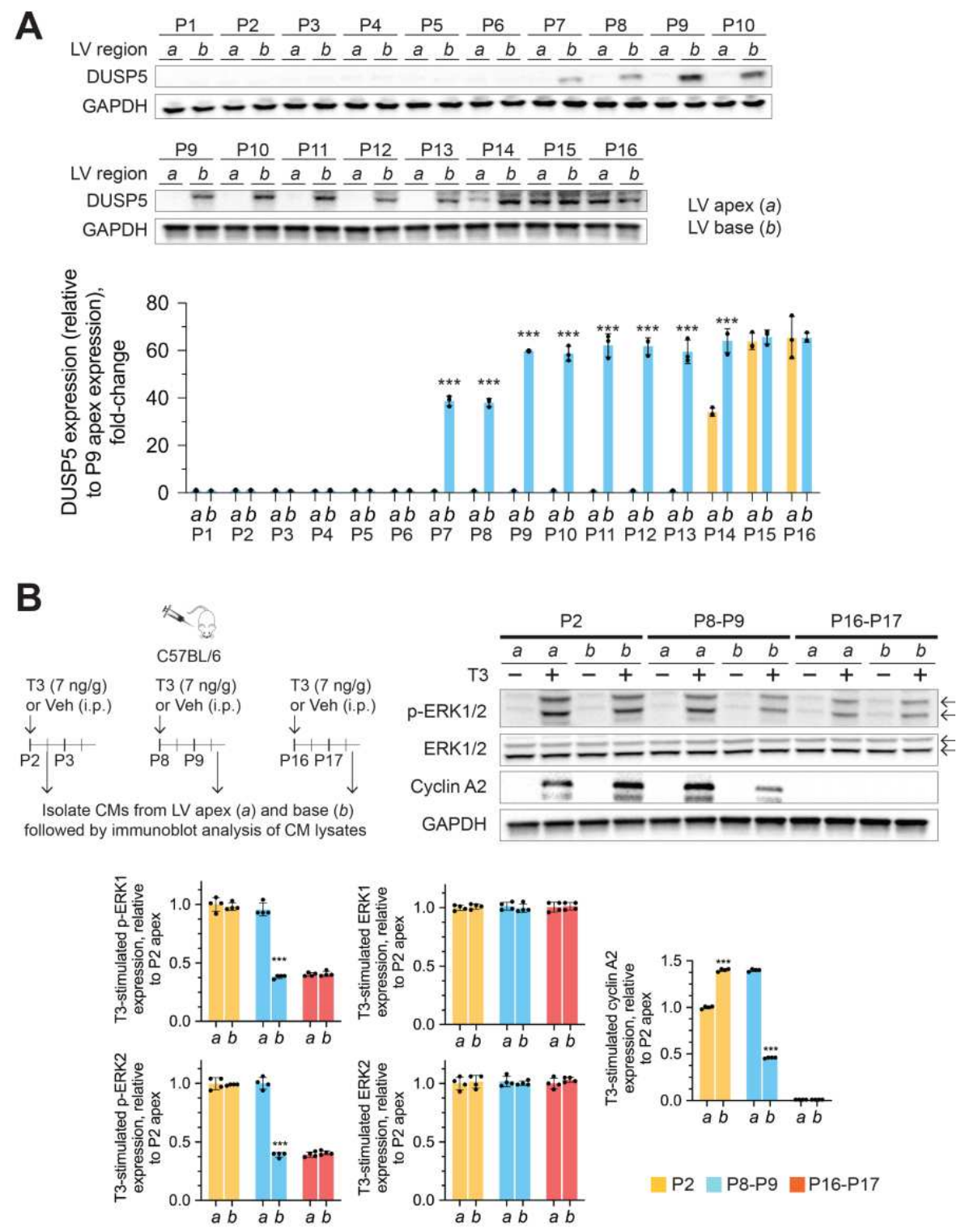

Figure 6. Spatio-temporal changes in LV DUSP5 levels and T3-stimulated ERK1/2 phosphorylation in cardiomyocytes in postnatal mice. (A) Representative immunoblots showing developmental changes in myocardial DUSP5 levels in the LV apex $(a)$ and base $(b)$ of early postnatal hearts. Bar graphs show GAPDH normalized quantitative data relative to DUSP5 levels in apical tissue of the P9 LV myocardium. (B) Representative immunoblots showing expression of the indicated proteins in whole cell lysates prepared from cardiomyocytes (CMs) of the LV apex and base of P2 (neonatal), P8 (early preadolescent) and P16 (preadolescent) hearts, both with and without in vivo T3 treatment. The upper and lower arrows indicate the position of ERK1 and 2, respectively. Bar graphs show T3 stimulated, GAPDH normalized, protein expression, relative to expression in $\mathrm{P} 2 \mathrm{LV}$ apex derived CMs. Immunoblots are representative of 3 (A) or 4 (B) biologically independent replicates. Schematics illustrate the experimental protocol. Data are means \pm SEM. Comparisons between apex and base LV tissue samples were made using a paired 2-tailed Student's $t$-test, with $\mathrm{n}=3$ or 4 pairs at each time point. 
Table 1. Cardiomyocyte Numbers, Body weights, LV mass and Dimensions and LV Contractile Function of 21-Day-Old C57BL/6 Wild-type and Dusp5-/- Mice

\begin{tabular}{lccc}
\hline & Wild-type & Dusp5-/ & $P$-value \\
\hline Mice, $n[\mathrm{M} / \mathrm{F}]$ & $8[6 / 2]$ & $8[4 / 4]$ & \\
Body weight, g & $11.4 \pm 0.54$ & $10.7 \pm 0.36$ & 0.36 \\
LV mass, mg & $39.1 \pm 1.6$ & $52.5 \pm 1.7$ & $<0.001$ \\
Cardiomyocyte & & & \\
numbers, (millions) & $2.36 \pm 0.045$ & $2.73 \pm 0.065$ & $<0.001$
\end{tabular}

LV short-axis parameters

LV mid-papillary level

$\begin{array}{lccc}\text { LVPW-d, mm } & 0.39 \pm 0.018 & 0.54 \pm 0.012 & <0.001 \\ \text { LVPW-s, mm } & 0.86 \pm 0.038 & 0.92 \pm 0.009 & 0.15 \\ \text { LVID-d, mm } & 3.22 \pm 0.06 & 2.87 \pm 0.087 & 0.005 \\ \text { LVID-s, mm } & 2.39 \pm 0.049 & 1.88 \pm 0.056 & <0.001 \\ \text { Fractional shortening, \% } & 25.9 \pm 1 & 34.2 \pm 1.24 & <0.001 \\ \text { LV mid-apical level } & & & \\ \text { LVPW-d, mm } & 0.34 \pm 0.013 & 0.53 \pm 0.017 & <0.001 \\ \text { LVPW-s, mm } & 0.85 \pm 0.035 & 0.93 \pm 0.016 & 0.048 \\ \text { LVID-d, mm } & 2.71 \pm 0.062 & 2.4 \pm 0.087 & 0.011 \\ \text { LVID-s, mm } & 1.88 \pm 0.049 & 1.47 \pm 0.036 & <0.001 \\ \text { Fractional shortening, \% } & 30.5 \pm 0.94 & 38.5 \pm 1.8 & 0.0013\end{array}$

LV long-axis parameters

$\begin{array}{lccc}\text { LVPW-d, mm } & 0.34 \pm 0.014 & 0.54 \pm 0.018 & <0.001 \\ \text { LVPW-s, mm } & 0.85 \pm 0.032 & 0.93 \pm 0.016 & 0.038 \\ \text { LVID-d, mm } & 4.29 \pm 0.058 & 4.52 \pm 0.052 & 0.0085 \\ \text { LVID-s, mm } & 3.49 \pm 0.066 & 3.46 \pm 0.047 & 0.47 \\ \text { Fractional shortening, \% } & 18.5 \pm 1.4 & 23.5 \pm 1 & 0.011 \\ & & & \\ \text { LV volume at diastole, } \mu \mathrm{l} & 42.1 \pm 2.1 & 34.5 \pm 2.2 & 0.026 \\ \text { LV volume at systole, } \mu 1 & 20 \pm 1.1 & 11.8 \pm 0.78 & <0.001 \\ \text { Ejection fraction, } \% & 52.4 \pm 1.4 & 65.5 \pm 1.6 & <0.001 \\ \text { Stroke volume, } \mu \mathrm{l} & 22.1 \pm 1.37 & 22.7 \pm 1.7 & 0.79\end{array}$

* C57BL/6 and Dusp5-/- mouse litters were adjusted at P2 to 4 pups/dam). Transthoracic echocardiography, M- and B-modes, were performed in 21-day-old mice under light isoflurane anesthesia. Immediately following this procedure, cardiomyocyte numbers were determined in whole-ventricle cell-suspensions of 
disaggregated hearts using a hemocytometer. Cardiomyocytes were identified by their shape and size. Data were analyzed by unpaired 2-tailed Students $t$-test. Values are mean \pm SEM. Abbreviations: left ventricle, LV; LV posterior wall dimension at diastole, LVPWD-d; LV posterior wall dimension at systole, LVPWD-s; LV internal dimension at end-diastolic, LVID-d; LV internal dimension at end-systole, LVID-s. 


\section{Figures}

A

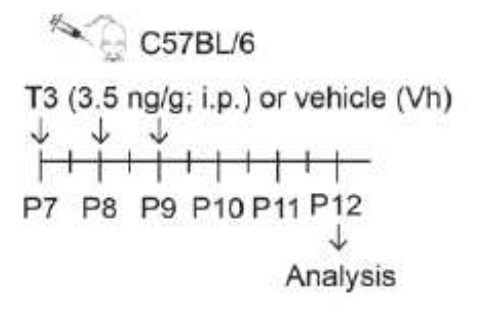

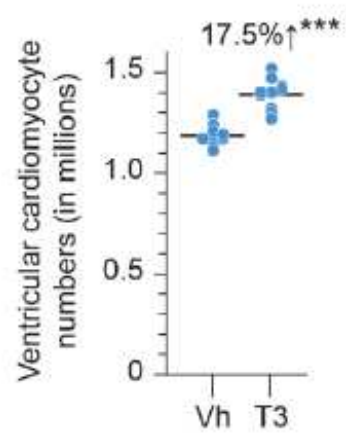

B

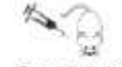

C57BL/6

T3 $(7 \mathrm{ng} / \mathrm{g})$

or Veh (i.p.)

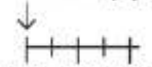

P8 P9P10

Analysis

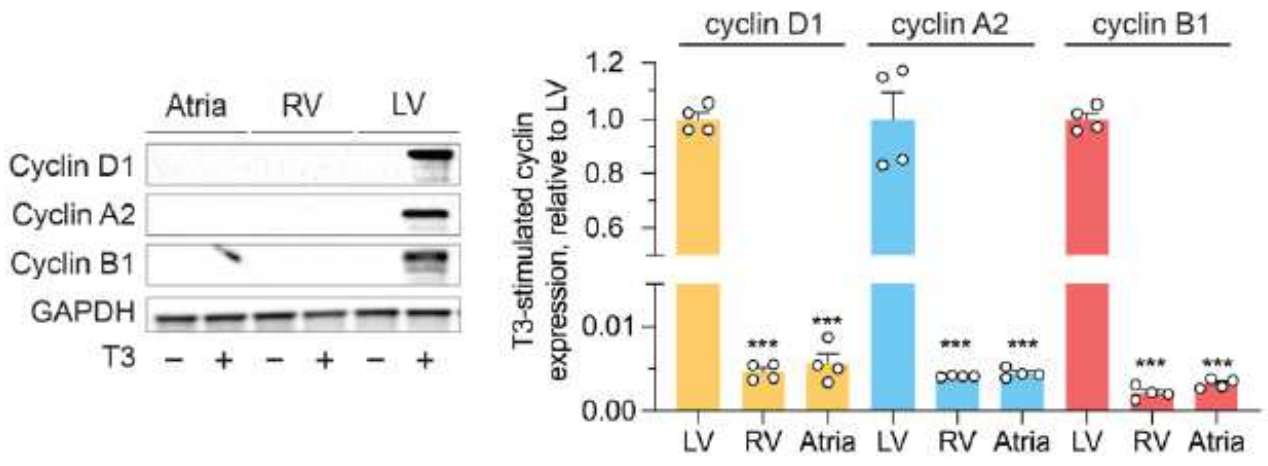

\section{C}

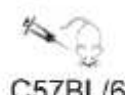

C57BL/6

T3 $(7 \mathrm{ng} / \mathrm{g})$

or Veh (i.p.)

$\downarrow$

P8 P9P10

Analysis
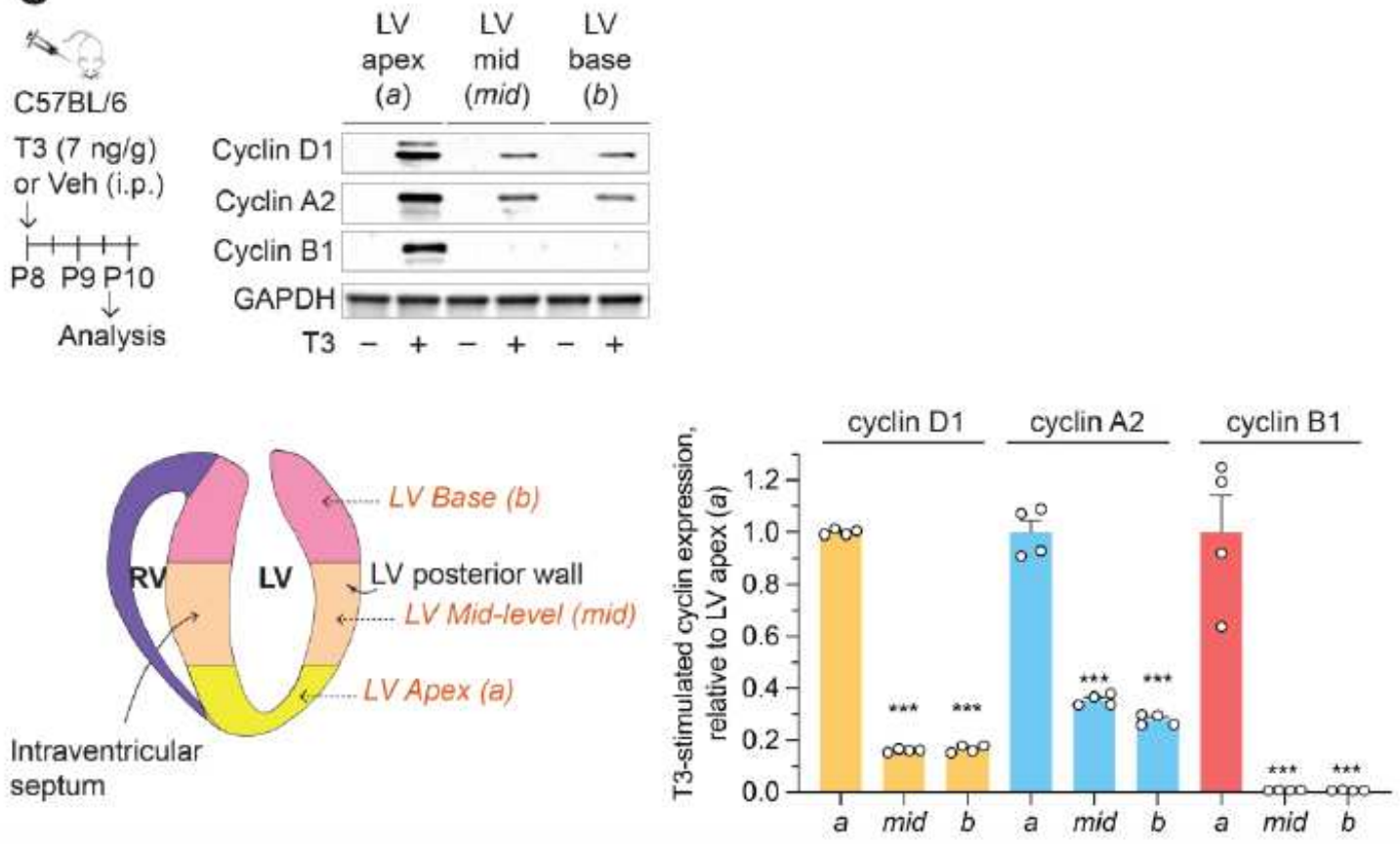

\section{Figure 1}

T3 increases ventricular cardiomyocyte proliferation and cyclin expression in the LV myocardium of postP6 hearts. (A) Exogenous T3 administration increases ventricular cardiomyocyte numbers in P7 mice. ${ }^{* \star *} \mathrm{P}<0.001$. Schematics illustrate experimental protocol. $(\mathrm{B}, \mathrm{C})$ In vivo T3 administration increases cyclin $D 1, A 2$, and $B 1$ expression in the LV, but not atria or right ventricle (RV) (B) and, within the LV, preferentially in the apex compared to the mid LV or base (C). Histograms show the quantitation of the data in (B) and 
(C). Immunoblots in B and C are representative of 4 biologically independent replicates. Schematics illustrate experimental protocol. Error bars indicate SEM. ${ }^{\star \star *} \mathrm{P}<0.001$.
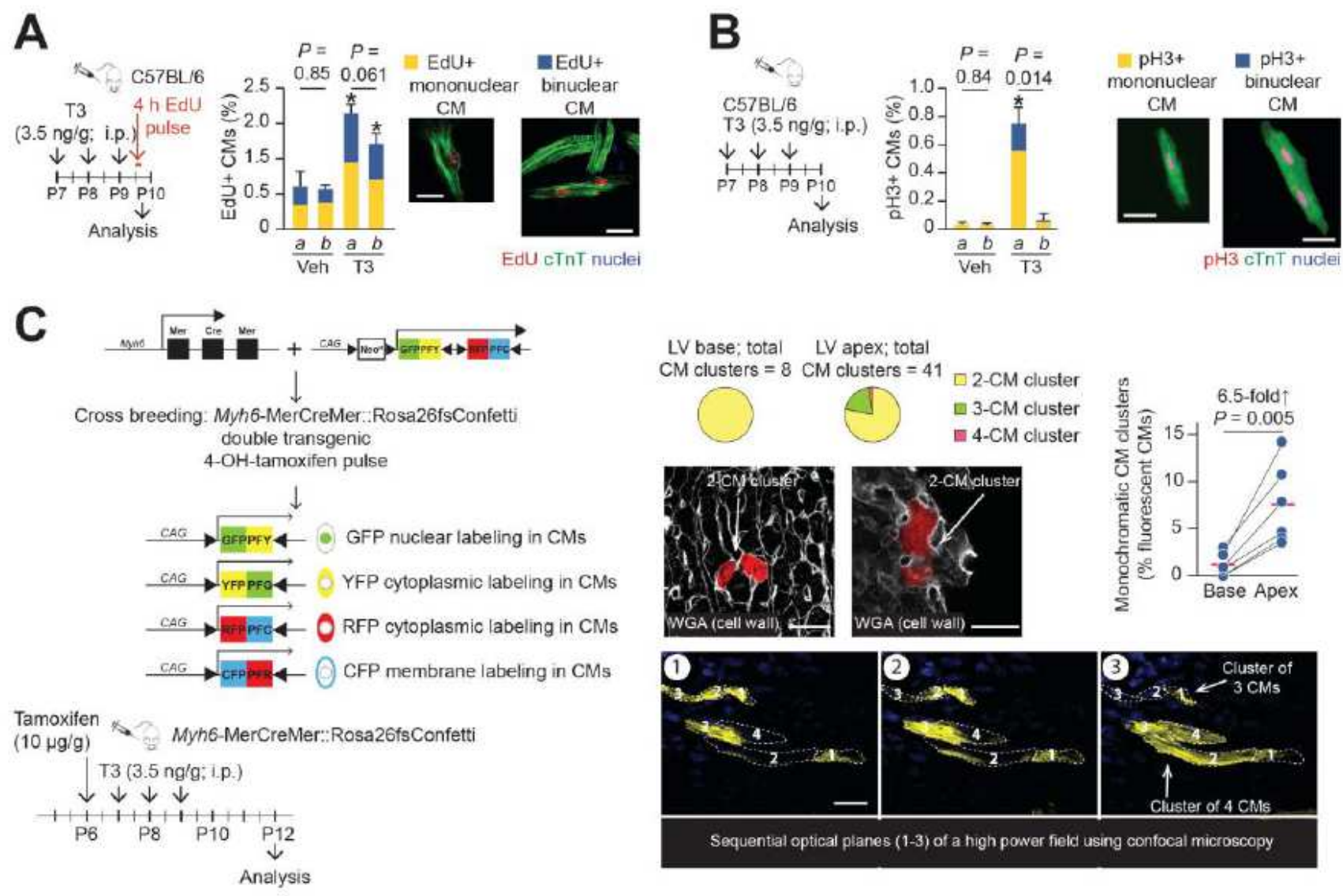

Figure 2

LV apical cardiomyocytes (CM) of post-P6 hearts proliferate in response to exogenous T3 versus vehicle. (A, B) Effects of T3 on DNA synthesis (EdU labeling) (A) and mitosis ( $\mathrm{pH}$ labeling) (B) in cardiomyocytes from LV apex (a) or base (b). Cardiomyocytes were identified by cTnT labeling. * $P<0.05$ for vehicle (Veh) versus T3 comparisons within each region using an unpaired Student's t-test; $n=4$. Intra-LV comparisons were made using a paired Student's t-test; P-values for these comparisons are indicated. Between 500 and 2,000 cardiomyocytes were analyzed for each assessment. (C) Multicolor labeling of post-neonatal mouse LV cardiomyocytes. Limited 4-hydroxytamoxifen-induced recombination of paired loxP sites (black triangles) leads to expression of green fluorescent protein (GFP), yellow fluorescent protein (YFP), red fluorescent protein (RFP) or cyan fluorescent protein (CFP) in a few cardiomyocytes, randomly positioned within the entire LV. Insets show examples of monochromatic clusters. Intra-LV comparisons were made using a paired Students t-test. Schematics illustrate experimental protocol. Error bars indicate SEM. White bars in photomicrographs represent $20 \mu \mathrm{m}$. 

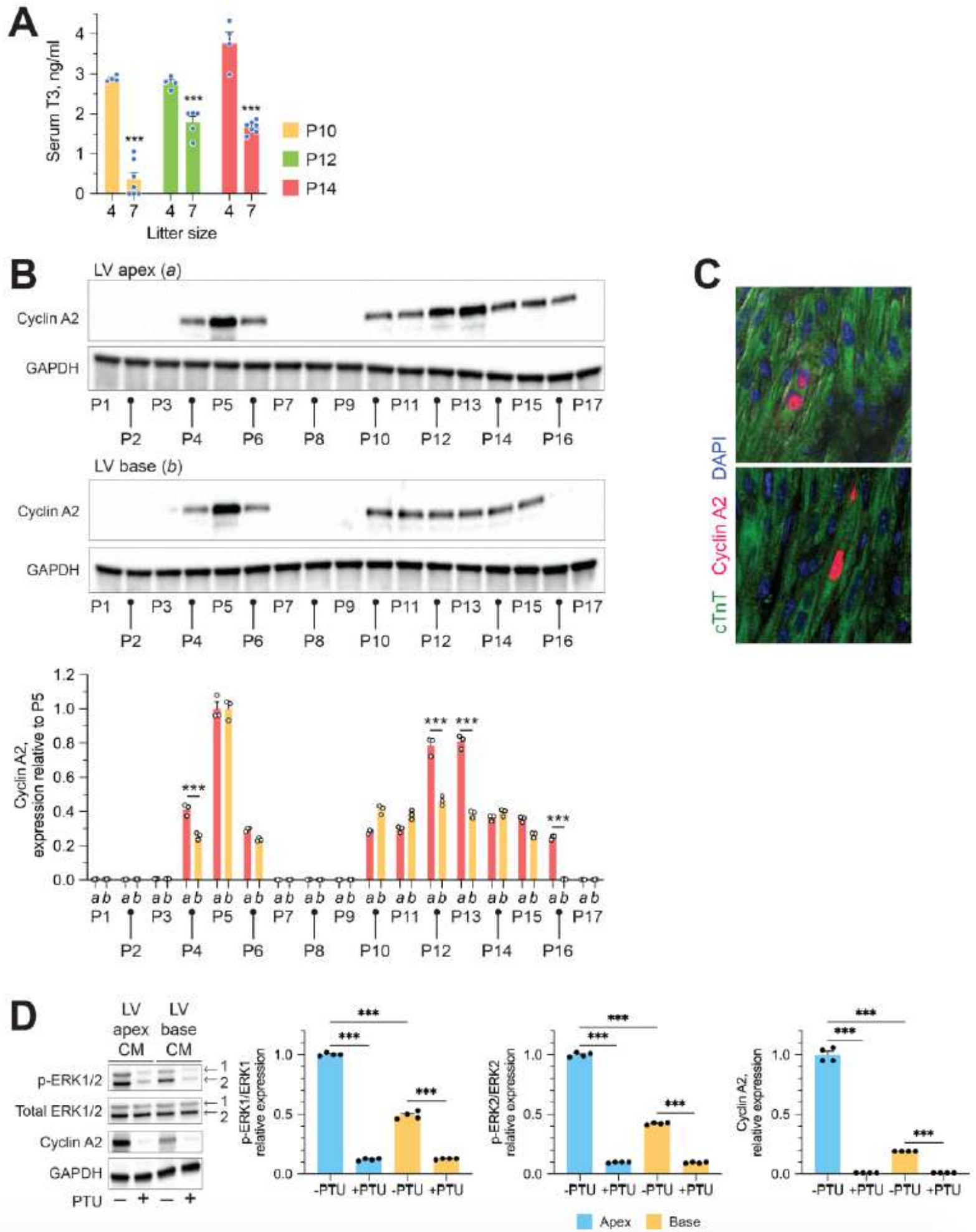

\section{Figure 3}

Spatial restriction of cardiomyocytes proliferative signaling to the LV apex during preadolescence. (A) Effect of litter size on developmental surge in circulating T3 during early preadolescence. (B) Representative immunoblots showing developmental changes in myocardial Cyclin A2 levels in the LV apex (a) and base (b) of early postnatal hearts. Bar graphs show GAPDH-normalized cyclin A2 levels relative to levels in P5 LV myocardium. (C) Two examples of a cyclin A2+ cardiomyocyte in a P13 LV 
apical tissue section. No cyclin A2+ cardiomyocytes were seen on sections of P13 LV base. (D) Representative immunoblots show expression levels of indicated proteins in whole cardiomyocyte (CM) lysates obtained from CMs of the LV apex and base at P13. Immunoblots show that developmental increases in p-ERK1/2 and Cyclin A2 in LV apical CMs of P13 mice are suppressed by PTU treatment that blocks T3 biosynthesis. Quantitative data is shown next to the immunoblots. ${ }^{\star} * * P 0.001$. Data are mean \pm SEM.

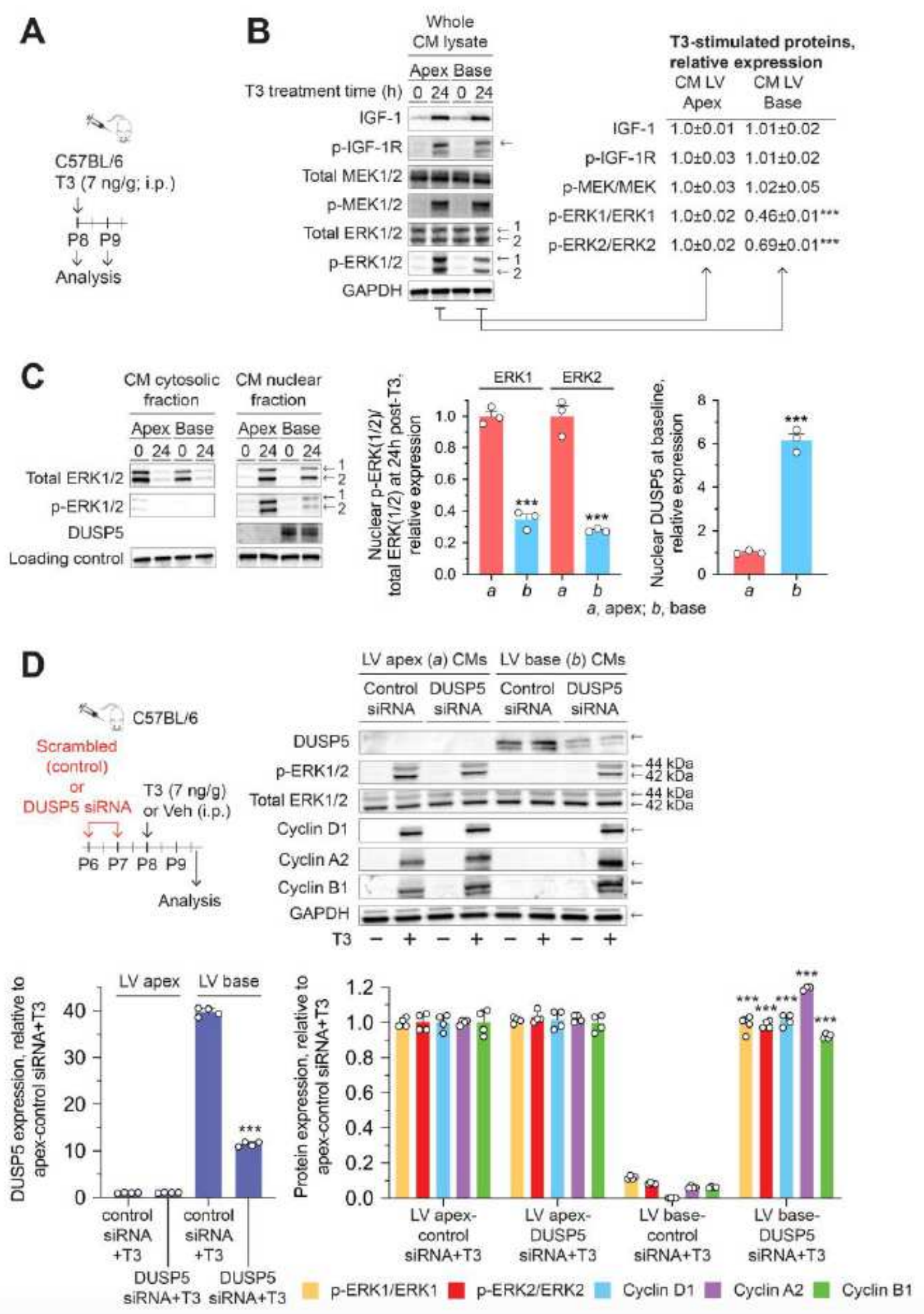


T3-induced ERK1/2 phosphorylation and cyclin expression in cardiomyocytes (CMs) of the P8 LV base is inhibited by DUSP5. (A) Schematic showing the protocol for T3 administration and analysis times. (B) Representative immunoblots show expression levels of indicated proteins in whole CM lysates obtained from CMs of the LV apex and base at 0 (baseline) and $24 \mathrm{~h}$ after T3 administration. Quantitative data relative to that obtained from analysis of CMs of the LV apex, are shown next to the immunoblots. (C) Using the same protocol as in A, isolated CMs were resolved into their constituent nuclear and cytosol fractions before immunoblot analysis. Quantitative data are shown in bar graphs adjacent to the immunoblots. In B and C, immunoblots are representative of 4 biologically independent replicates. (D) DUSP5 or scrambled (control) siRNA was administered intraperitoneally (i.p.) to mice at P6 and P7, followed by T3 $(+)$ or vehicle $(-)$ treatment at P8. siRNAs were dissolved in the in vivo-jetPEl and $10 \%$ glucose mixture. Forty hours later, CMs were isolated and whole cell lysates prepared. Lysates were then subjected to immunoblotting for the indicated proteins. Immunoblots are representative of 4 biologically independent replicates. Quantitative data are presented in the histograms. Data are mean \pm SEM. ${ }^{\star \star \star} \mathrm{P}<$ 0.001 comparisons for intra-LV region derived cardiomyocytes from control versus DUSP5 siRNA treated mice. 


\section{Cardiomyocytes of the P7-P8 LV base}
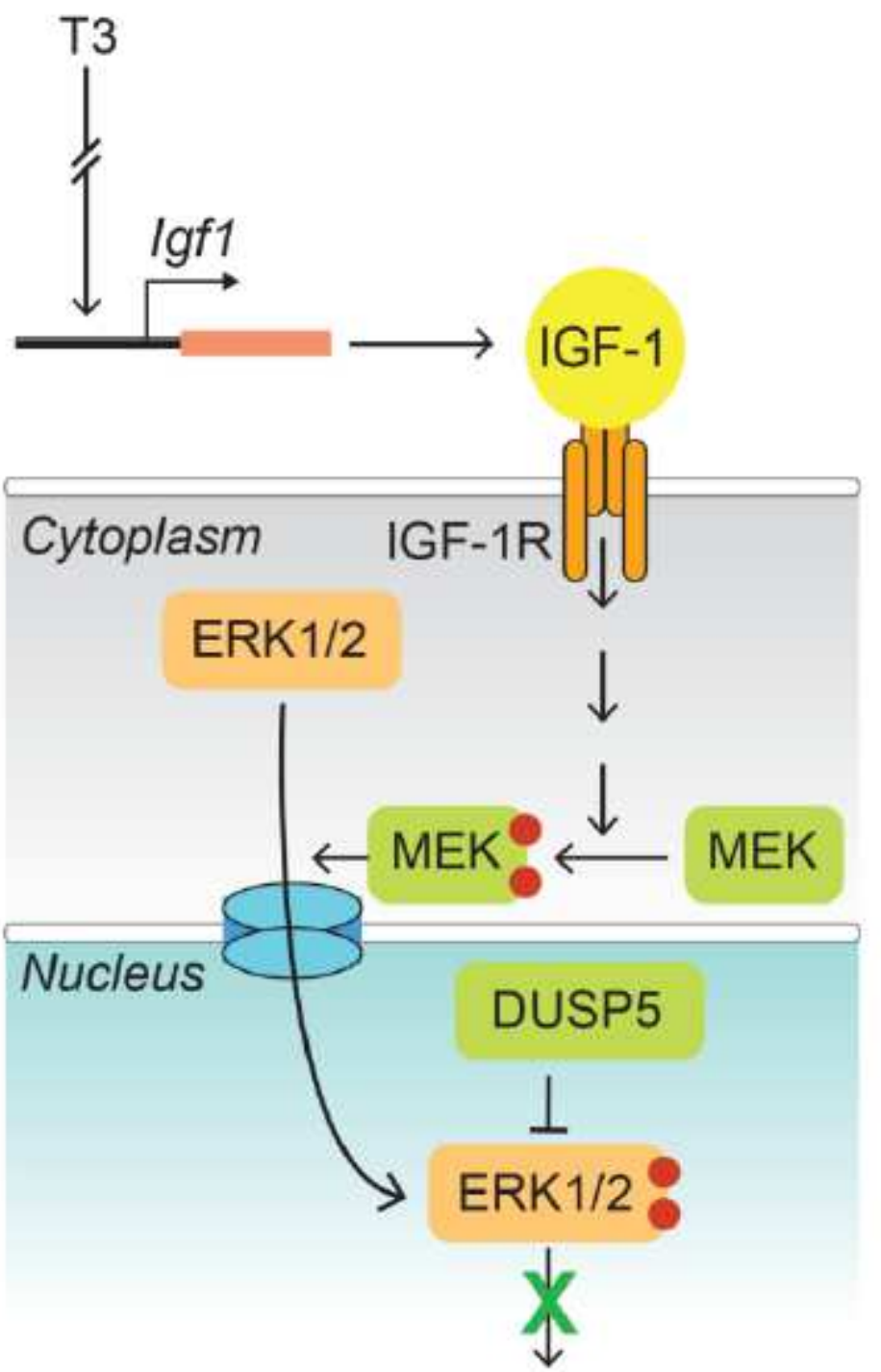

Proliferation

- Phosphorylation

Figure 5

A working model illustrating the mechanism underlying the selective loss of nuclear phosphorylated ERK $1 / 2$ in cardiomyocytes from the base of the LV of post-P6 mice. 

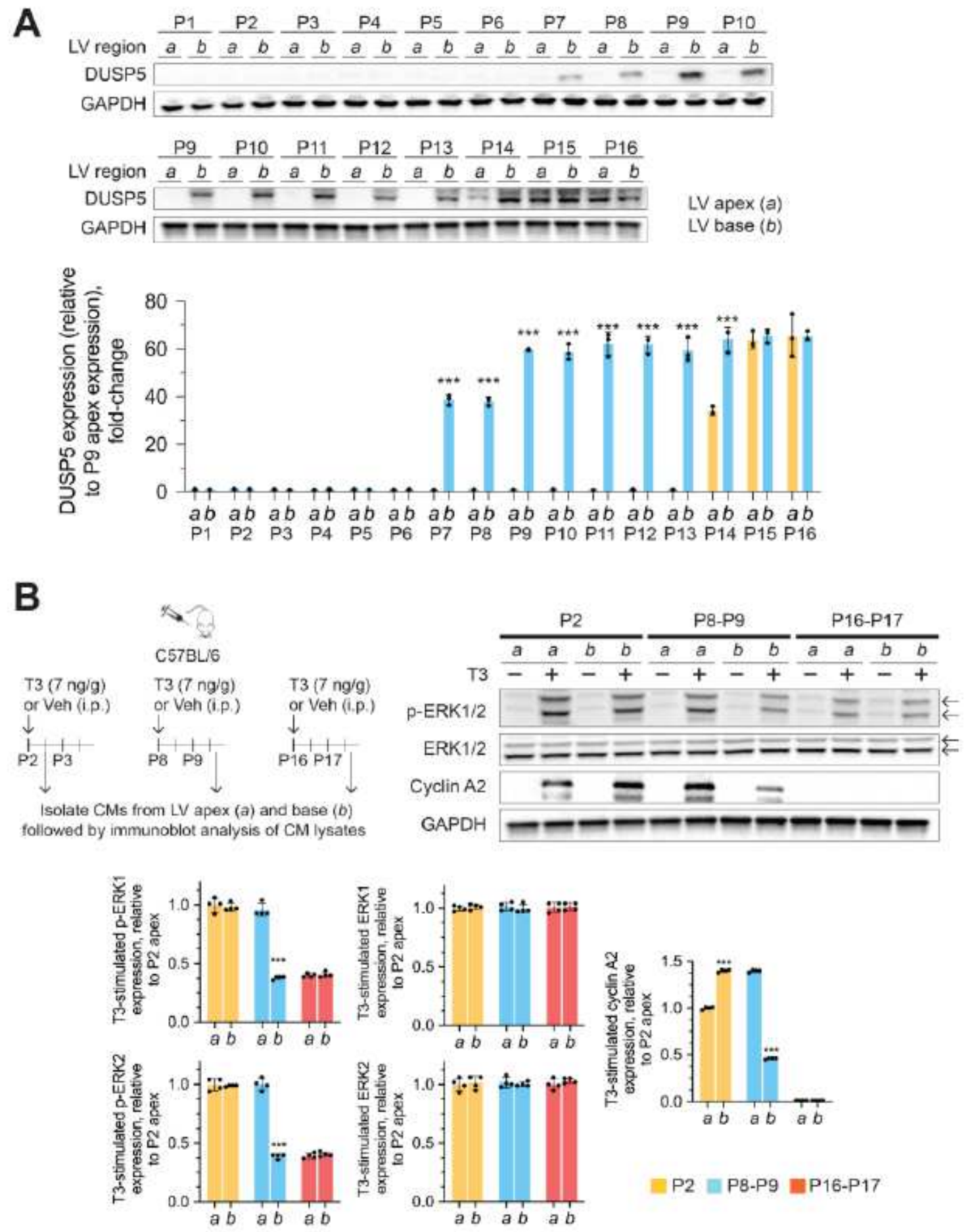

P2 핌 P8-P9 $=\mathrm{P} 16-\mathrm{P} 17$

\section{Figure 6}

Spatio-temporal changes in LV DUSP5 levels and T3-stimulated ERK1/2 phosphorylation in cardiomyocytes in postnatal mice. (A) Representative immunoblots showing developmental changes in myocardial DUSP5 levels in the LV apex (a) and base (b) of early postnatal hearts. Bar graphs show GAPDH normalized quantitative data relative to DUSP5 levels in apical tissue of the P9 LV myocardium. (B) Representative immunoblots showing expression of the indicated proteins in whole cell lysates 
prepared from cardiomyocytes (CMs) of the LV apex and base of P2 (neonatal), P8 (early preadolescent) and P16 (preadolescent) hearts, both with and without in vivo T3 treatment. The upper and lower arrows indicate the position of ERK1 and 2, respectively. Bar graphs show T3 stimulated, GAPDH normalized, protein expression, relative to expression in P2 LV apex derived CMs. Immunoblots are representative of 3 (A) or 4 (B) biologically independent replicates. Schematics illustrate the experimental protocol. Data are means \pm SEM. Comparisons between apex and base LV tissue samples were made using a paired 2tailed Student's t-test, with $\mathrm{n}=3$ or 4 pairs at each time point.

\section{Supplementary Files}

This is a list of supplementary files associated with this preprint. Click to download.

- BogushSCIREPJune2020supplement.pdf 